\title{
Synthesis of 3-Alkyl Enol Mimics Inhibitors of Type II Dehydroquinase: Factors Influencing Their Inhibition Potency
}

\author{
Beatriz Blanco, ${ }^{a}$ Antía Sedes, ${ }^{a}$ Antonio Peón, ${ }^{a}$ Heather Lamb, ${ }^{b}$ Alastair R. Hawkins, ${ }^{b}$ Luis Castedo ${ }^{c}$ and \\ Concepción González-Bello $*^{a}$
}

\author{
s Received (in $X X X, X X X)$ Xth $X X X X X X X X X 20 X X$, Accepted Xth $X X X X X X X X X 20 X X$ \\ DOI: $10.1039 / b 000000 x$
}

Several 3-alkylaryl mimics of the enol intermediate in the reaction catalyzed by type II dehydroquinase were synthesized to investigate the effect in the inhibition potency of replacing the oxygen atom in the side chain by a carbon atom. The length and the ridigity of the spacer was also studied. The inhibitory

10 properties of the resported compounds against type II dehydroquinase from Mycobacterium tuberculosis and Helicobacter pylori are also reported. The binding modes of these analogs in the active site of both enzymes were studied by molecular docking using GOLD 5.0 and dynamic simulations studies.

\section{Introduction}

In recent years, we have been working on the development of 15 new antibiotics for the treatment of bacterial infections, ${ }^{[1]}$ by inhibition of type II dehydroquinase (DHQ2), which catalyzes the reversible dehydration of 3-dehydroquinic acid (1) to form 3dehydroshikimic acid (2) (Scheme 1). ${ }^{[2,3]}$ The reaction proceeds through an enol intermediate $\mathbf{3}$, which is stabilized by a 20 conserved water molecule that interacts through hydrogen bonding to Asn12, the carbonyl group of Pro11, and the mainchain amide of Gly78. The final step is the acid-catalyzed elimination of the C- 1 hydroxyl group - a reaction mediated by a histidine residue, which acts as a proton donor. ${ }^{[4]}$
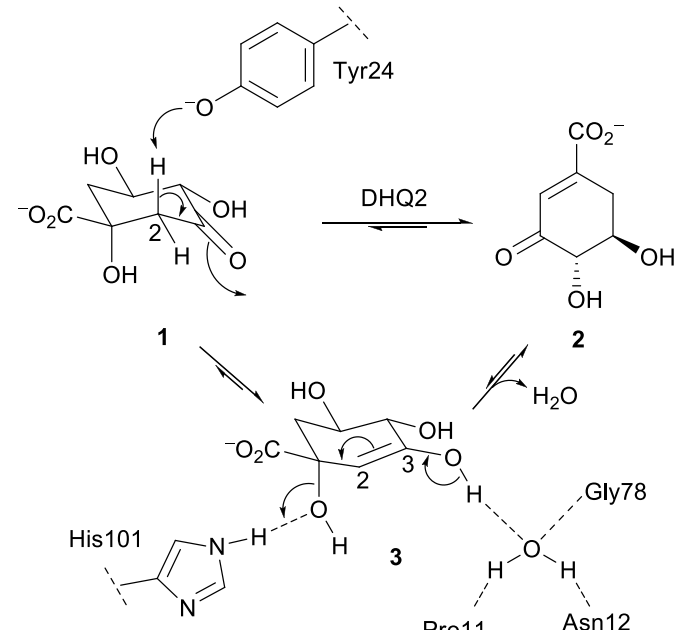

25

Scheme 1. Enzymatic conversion of 3-dehydroquinic acid (1) to 3-dehydroshikimic acid (2) catalyzed by DHQ2. The reaction proceeds via an enol intermediate 3. Relevant residues are indicated (the numbering corresponds to M. tuberculosis).

30 In particular, we have focused on the inhibition of two pathogenic bacteria, Mycobacterium tuberculosis, the causative agent of tuberculosis and Helicobacter pylori, the causative agent of gastric and duodenal ulcers, which has also been classified as a type I carcinogen. We recently showed that 3-methoxyaryl 35 derivatives $\mathbf{4 a}-\mathbf{c}$ (Figure 1), in which the aryl moiety is linked to the cyclohexene core by a methoxy group, are potent competitive inhibitors of DHQ2 from Helicobacter pylori (DHQ2-Hp) and Mycobacterium tuberculosis (DHQ2-Mt). ${ }^{[5]}$

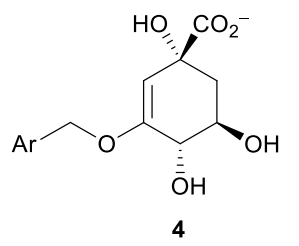

$\operatorname{Ar}=$

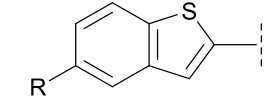

$4 \mathrm{~b} \mathrm{R}=\mathrm{H}, K_{\mathrm{i}}=28 \mathrm{nM}$

$4 \mathrm{c} \mathrm{R}=\mathrm{Me}, K_{\mathrm{i}}=42 \mathrm{nM}$
40 Figure 1. Selected examples of 3-methoxyaryl derivatives that are DHQ2 competitive inhibitors. Inhibition constants against DHQ2-Mt are indicated.

The crystal structures of DHQ2-Hp and DHQ2-Mt in complex with compound $\mathbf{4 c}$ have been solved at $2.95 \AA$ and $1.5 \AA$, 45 respectively (Figure 2). ${ }^{[5,6]}$ These crystal structures clarified the role of the aromatic rings on $\mathrm{C} 3$, which block the entrance of the essential arginine side chain into the active site and cause an important change in the conformation and flexibility of the loop that closes over the substrate binding site. Molecular dynamics 50 simulation studies suggest that the aromatic ring prevents appropriate orientation of the catalytic tyrosine of the loop for proton abstraction and disrupts its basicity. ${ }^{[7]}$ The crystal structure solved at $1.5 \AA$ shows that the oxygen atom of the methylenoxy spacer of the inhibitor $\mathbf{4 c}$ is located $3.1 \AA$ away from the 55 conserved water molecule involved in the catalysis (Figure $2 b$ ). We assume that an important contribution of the high potency of the inhibitor, with $K_{\mathrm{i}}$ values of $42 \mathrm{nM}^{[5 \mathrm{~b}]}$ and $130 \mathrm{nM}^{[5 \mathrm{a}]}$ against DHQ2-Mt and DHQ2-Hp, respectively, is due to the hydrogen- 
bonding interaction between the oxygen atom of the methylenoxy spacer with the conserved water molecule. In order to corroborate this hypothesis, we decided to investigate the effect in the inhibition potency of replacing the oxygen atom in the side chain 5 of $\mathbf{4} \mathbf{a}-\mathbf{b}$ by a carbon atom. In addition, the length and the rigidity of the alkylene spacer was also studied. To this end, 3-alkylaryl enol mimics 5, 6 and 7, having a vinylene, ethylene and propylene spacer, respectively, were designed (Figure 3). The results of inhibition studies of these compounds against DHQ2${ }_{10} \mathrm{Mt}$ and DHQ2-Hp, docking studies using GOLD 5.0 and dynamic simulations studies are also described.
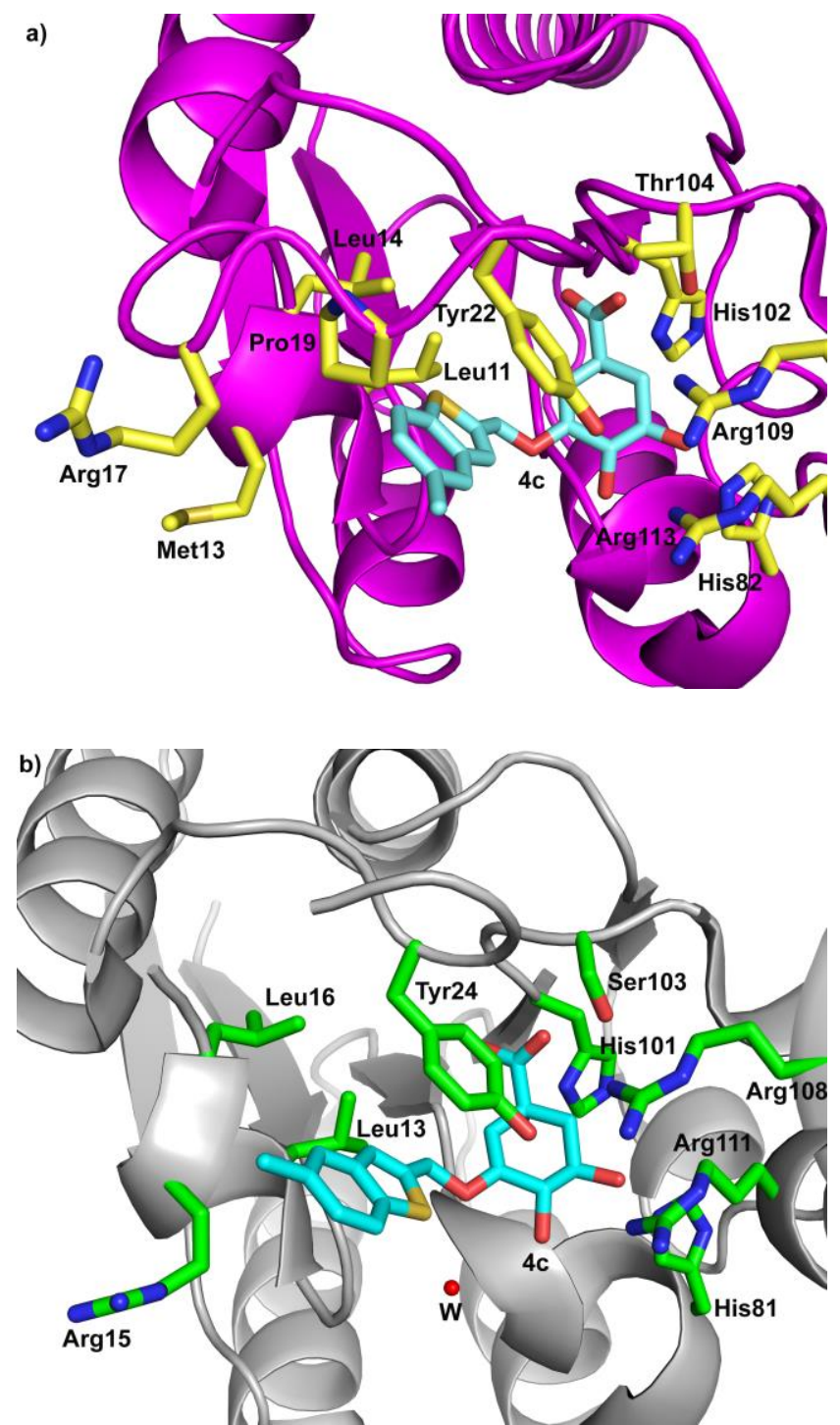

Figure 2. Selected views of the crystal structures of the binary complex of: a) $15 \mathrm{DHQ} 2-\mathrm{Hp} / 4 \mathrm{c}$ (PDB: 2WKS, $2.95 \AA)^{[5 \mathrm{a}]}$; b) DHQ2-Mt/4c (PDB: 2 Y71, $\left.1.5 \AA\right)^{[5 b]}$. Relevant residues are indicated.

\section{Results and Discussion}

\section{Synthesis of vinylene derivatives 5}

The synthesis of the target compounds $\mathbf{5}$ was achieved by Suzuki 20 cross-coupling reactions between our previously reported vinyl triflate $\mathbf{1 2}^{[2 \mathrm{c}]}$ and the appropriate boronic acid pinacol esters $\mathbf{1 1}$ (Scheme 2). Firstly, the Sonogashira cross-coupling reaction of commercially available aryl bromides $\mathbf{8}$ with trimethylsilylacetylene gave the protected alkynes $\mathbf{9}$, which by 25 deprotection with TBAF afforded terminal alkynes $\mathbf{1 0}$ (Scheme 2 and Table 1). Finally, hydroboration of alkynes $\mathbf{1 0}$ with catechol borane gave the required boronic acid pinacol esters $\mathbf{1 1}$ in good yield. Suzuki cross-coupling between vinyl triflate $\mathbf{1 2}^{[2 \mathrm{c}]}$ and boronic acid pinacol esters 11 gave the corresponding cross30 coupling products $\mathbf{1 3}$, which were converted to the desired acids 5 by deprotection followed by basic hydrolysis of the corresponding lactones $\mathbf{1 4}$ and protonation with an ion-exchange resin.<smiles>[R]C1=C[C@@](O)(C(=O)O)C[C@@H](O)[C@@H]1O</smiles>

$5 \mathrm{R}=(E)-\mathrm{CH}=\mathrm{CH}$

$6 \mathrm{R}=\left(\mathrm{CH}_{2}\right)_{2}$ $7 \mathrm{R}=\left(\mathrm{CH}_{2}\right)_{3}$

35 Figure 3. Target compounds
$\mathrm{Ar}=$ naphth-2-y

b $\mathrm{Ar}=$ benzo[b]thiophen-2-yl c $\mathrm{Ar}=5,6,7,8$-tetrahydronaphth-2-yl d $\mathrm{Ar}=$ benzo[b]thiophen-3-yl
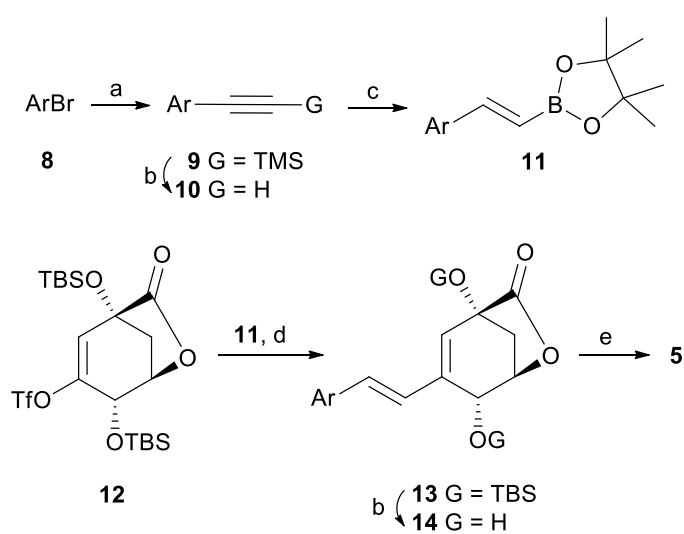

Scheme 2. Synthesis of compounds 5. Reagents and conditions: a) HCCTMS, CuI, $\left.\left.\mathrm{Pd}\left(\mathrm{PPh}_{3}\right)_{2} \mathrm{Cl}_{2}, \mathrm{Et}_{3} \mathrm{~N}, 40{ }^{\circ} \mathrm{C} ; \mathrm{b}\right) \mathrm{TBAF}, \mathrm{THF}, \mathrm{RT} ; \mathrm{c}\right) 1$. Catechol borane, THF, $\Delta ; 2$. Pinacol, THF, $\Delta$; d) $\mathrm{Pd}\left(\mathrm{PPh}_{3}\right)_{4}, \mathrm{~K}_{3} \mathrm{PO}_{4}$ (aq.), dioxane, $80{ }^{\circ} \mathrm{C}$; e) $1 . \mathrm{LiOH}, \mathrm{THF}, \mathrm{RT}$; 40 2. Amberlite IR-120 $\left(\mathrm{H}^{+}\right)$.

Table 1. Synthesis of compounds 9-11, 13, 14 and $\mathbf{5}^{a}$

\begin{tabular}{ccccc}
\hline Reaction & Comp & Yield (\%) & Comp & Yield (\%) \\
\hline $\mathbf{8} \rightarrow \mathbf{9}$ & $\mathbf{9 a}$ & 99 & $\mathbf{9 b}$ & 98 \\
$\mathbf{9} \rightarrow \mathbf{1 0}$ & $\mathbf{1 0 a}$ & 98 & $\mathbf{1 0 b}$ & 87 \\
$\mathbf{1 0} \rightarrow \mathbf{1 1}$ & $\mathbf{1 1 a}$ & 85 & $\mathbf{1 1 b}$ & 94 \\
$\mathbf{1 2} \rightarrow \mathbf{1 3}$ & $\mathbf{1 3 a}$ & 94 & $\mathbf{1 3 b}$ & 87 \\
$\mathbf{1 3} \rightarrow \mathbf{1 4}$ & $\mathbf{1 4 a}$ & 65 & $\mathbf{1 4 b}$ & 43 \\
$\mathbf{1 4} \rightarrow \mathbf{5}$ & $\mathbf{5 a}$ & 77 & $\mathbf{5 b}$ & 79 \\
\hline \multicolumn{5}{c}{$\mathbf{a}$ Ar $=$ naphth-2-yl; $\mathbf{b}$ Ar = benzo $[b]$ thiophen-2-yl. }
\end{tabular}

\section{${ }_{45}$ Synthesis of ethylene derivatives 6}

The synthesis of ethylene side-chain acids $\mathbf{6}$ was first addressed by selective reduction of the external double bond in dienes $\mathbf{1 3}$ (Scheme 3 and Table 2). Catalytic hydrogenation of 13 using Rosemund's catalyst gave the desired saturated derivatives 15a 
and $\mathbf{1 5 b}$ in $75 \%$ and $56 \%$ yield, respectively. Surprisingly, the reduction of naphthyl derivative $\mathbf{1 3 a}$ also afforded a $20 \%$ yield of compound $15 \mathbf{c}$ resulting from a partial reduction of the naphthyl moiety. However, this side reduction was avoided by using 5 Raney-Ni as catalyst to afford compound 15a as a single product in $78 \%$ yield. The tetrahydronaphthyl derivative $\mathbf{1 5 c}$ was also transformed into its corresponding acid $\mathbf{6 c}$ to test its biological activity.
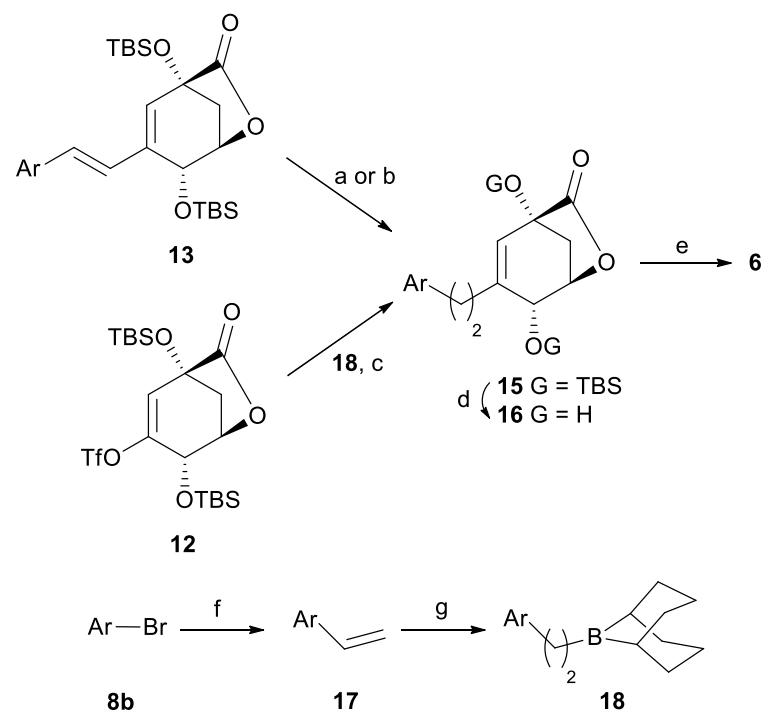

10 Scheme 3. Synthesis of acids 6. Reagents and conditions: a) $\mathrm{H}_{2}$, Rosemund's catalyst, $50 \%$ THF/MeOH, RT; b) $\mathrm{H}_{2}$, Raney-Ni, 50\% THF/MeOH, RT; c) $\mathrm{PdCl}_{2}(\mathrm{dppf}), \mathrm{K}_{3} \mathrm{PO}_{4}$, THF, $\Delta$; d) TBAF, THF, RT; e) 1. LiOH, THF, RT; 2. Amberlite IR-120 ( $\left.\mathrm{H}^{+}\right)$; f) vinyl boronic acid pinacol ester, $\mathrm{Pd}\left(\mathrm{PPh}_{3}\right)_{4}, \mathrm{~K}_{3} \mathrm{PO}_{4}$ (aq.), dioxane, $80^{\circ} \mathrm{C}$; g) 9-BBN-H, THF, $0{ }^{\circ} \mathrm{C}$ to RT.

Table 2. Synthesis of compounds 15-18 and $6{ }^{a}$

\begin{tabular}{ccccccc}
\hline Reaction & Comp & $\begin{array}{c}\text { Yield } \\
(\%)\end{array}$ & Comp & $\begin{array}{c}\text { Yield } \\
(\%)\end{array}$ & Comp & $\begin{array}{c}\text { Yield } \\
(\%)\end{array}$ \\
\hline $\mathbf{1 3} \rightarrow \mathbf{1 5}$ & $\mathbf{1 5 a}$ & 75 & $\mathbf{1 5 b}$ & 56 & $\mathbf{1 5 c}$ & 20 \\
$\mathbf{1 3 a} \rightarrow \mathbf{1 5}$ & $\mathbf{1 5 a}$ & 78 & -- & -- & $\mathbf{1 5 c}$ & 0 \\
$\mathbf{1 2} \rightarrow \mathbf{1 5}$ & $\mathbf{1 5 a}$ & 80 & $\mathbf{1 5 b}$ & 80 & $\mathbf{- -}$ & $\mathbf{-}$ \\
$\mathbf{1 5} \rightarrow \mathbf{1 6}$ & $\mathbf{1 6 a}$ & 79 & $\mathbf{1 6 b}$ & 60 & $\mathbf{1 6 c}$ & 90 \\
$\mathbf{1 6} \rightarrow \mathbf{6}$ & $\mathbf{6 a}$ & 85 & $\mathbf{6 b}$ & 83 & $\mathbf{6 c}$ & 85 \\
$\mathbf{8 b \rightarrow \mathbf { 1 7 b }}$ & $\mathbf{- -}$ & -- & $\mathbf{1 7 b}$ & 96 & $\mathbf{- -}$ & -- \\
\hline
\end{tabular}

${ }^{a} \mathbf{a ~ A r}=$ naphth-2-yl; $\mathbf{b} \mathrm{Ar}=$ benzo[b]thiophen-2-yl; $\mathbf{c} \mathrm{Ar}=5,6,7,8$-tetrahydronaphth2-yl.

20 The selective reduction of dienes $\mathbf{1 5}$ proved to be experimentally problematical due to the difficulty in controlling and monitoring the reduction. Because of that, we were particularly interested in addressing the synthesis of the alkyl lactones 15 by a direct $\mathrm{sp}^{3}-\mathrm{sp}^{2}$ cross-coupling reaction. After 25 numerous attempts using various $\mathrm{sp}^{3}$ boronic acids or their corresponding boronic acids pinacol esters, the cross-coupling was achieved by using alkyl boranes $\mathbf{1 8}$ and $\mathrm{PdCl}_{2}(\mathrm{dppf})$ as catalyst in the presence of $\mathrm{K}_{3} \mathrm{PO}_{4}$ in THF. ${ }^{[8]}$ Alkyl boranes $\mathbf{1 8}$ were synthesized by hydroboration with $9-\mathrm{BBN}-\mathrm{H}$ of vinyl 30 derivatives $\mathbf{1 7}$. Non-commercially available vinyl derivative $\mathbf{1 7} \mathbf{b}$ was prepared by Suzuki cross-coupling of halide $\mathbf{8 b}$ and vinyl boronic acid pinacol ester. Finally, compounds $\mathbf{1 5}$ were converted to the desired acids $\mathbf{6}$ in the same way as acids $\mathbf{5}$ from lactones 13.

35

\section{Synthesis of propylene derivatives 7}

Our initial attempts to synthesize compounds 7 involved as the key step the Sonogashira cross-coupling between the triflate $\mathbf{1 2}^{[2 \mathrm{c}]}$ and the terminal alkynes $\mathbf{2 0}$, followed by selective reduction of 40 the resulting enynes (Scheme 4 and Table 3 ). The required alkynes 20 were prepared by treatment of the Grignard derivative of $\mathbf{8}$ with (3-bromoprop-2-ynyl)trimethylsilane followed by deprotection. The latter reaction was achieved by treatment with $\mathrm{AgNO}_{3}$ in ethanol as the usual TBAF or $\mathrm{MeOH} / \mathrm{K}_{2} \mathrm{CO}_{3}$ 45 conditions afforded allenes $\mathbf{2 1}$ in good yield.
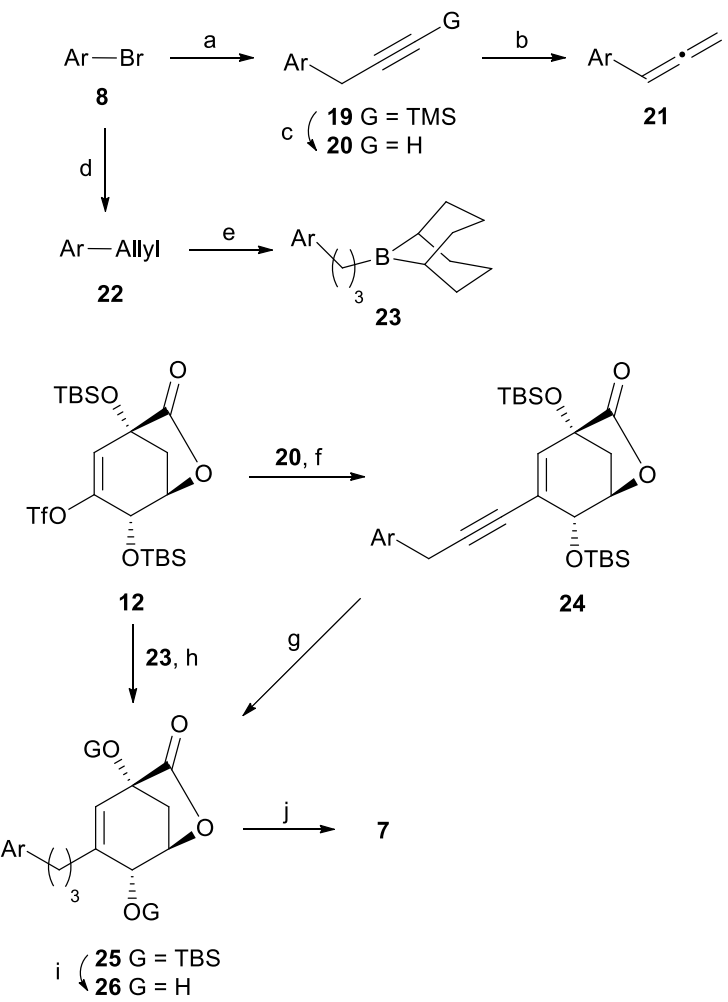

Scheme 4. Synthesis of compounds 7. Reagents and conditions: a) 1) $\mathrm{Mg}, \mathrm{I}_{2}$ (cat), $\mathrm{THF}, \Delta .2) \mathrm{TMSC} \equiv \mathrm{CCH}_{2} \mathrm{Br}$; b) $\mathrm{K}_{2} \mathrm{CO}_{3}, \mathrm{MeOH}, 0{ }^{\circ} \mathrm{C}$ to RT; c) $\mathrm{AgNO}_{3}$, EtOH (aq), RT; d) 1) $\mathrm{Mg}, \mathrm{I}_{2}$ (cat), THF, $\Delta$. 2) AllylBr; e) 9-BBN-H, THF, $0{ }^{\circ} \mathrm{C}$ to RT; f) $50 \mathrm{Pd}\left(\mathrm{PPh}_{3}\right)_{4}$, piperidine, CuI, THF, $\left.40{ }^{\circ} \mathrm{C} ; \mathrm{g}\right) \mathrm{H}_{2}$, Rosemund's catalyst, $50 \%$ THF/MeOH, RT; h) $\mathrm{PdCl}_{2}$ (dppf), $\mathrm{K}_{3} \mathrm{PO}_{4}$, THF, $\Delta$; i) TBAF, THF, RT; j) 1. LiOH, THF, RT; 2. Amberlite IR-120 $\left(\mathrm{H}^{+}\right)$.

Table 3. Synthesis of compounds 19-26 and 7.

\begin{tabular}{ccccc}
\hline Reaction & Comp & Yield (\%) & Comp & Yield (\%) \\
\hline $\mathbf{8} \rightarrow \mathbf{1 9}$ & $\mathbf{1 9 a}$ & 54 & $\mathbf{1 9 d}$ & 69 \\
$\mathbf{1 9 a} \rightarrow \mathbf{2 1 a}$ & $\mathbf{2 1 a}$ & 91 & -- & -- \\
$\mathbf{1 9 \rightarrow 2 0}$ & $\mathbf{2 0 a}$ & 72 & $\mathbf{2 0 d}$ & 61 \\
$\mathbf{8} \rightarrow \mathbf{2 2}$ & $\mathbf{2 2 a}$ & 99 & $\mathbf{2 2 d}$ & 89 \\
$\mathbf{1 2} \rightarrow \mathbf{2 4}$ & $\mathbf{2 4 a}$ & 98 & $\mathbf{2 4 d}$ & 95 \\
$\mathbf{2 4} \rightarrow \mathbf{2 5}$ & $\mathbf{2 5 a}$ & 98 & $\mathbf{2 5 d}$ & 98 \\
$\mathbf{1 2} \rightarrow \mathbf{2 5}$ & $\mathbf{2 5 a}$ & 70 & $\mathbf{2 5 d}$ & 42
\end{tabular}




\begin{tabular}{ccccc}
$\mathbf{2 5} \rightarrow \mathbf{2 6}$ & $\mathbf{2 6 a}$ & 77 & $\mathbf{2 6 d}$ & 67 \\
$\mathbf{2 6 \rightarrow 7}$ & $\mathbf{7 a}$ & 94 & $\mathbf{7 d}$ & 87 \\
\hline
\end{tabular}

${ }^{a} \mathbf{a} \mathrm{Ar}=$ naphth-2-yl; d Ar $=$ benzo[b]thiophen-3-yl.

Sonogashira cross-coupling reaction between terminal alkynes $\mathbf{2 0}$ and triflate $\mathbf{1 2}^{[2 \mathrm{c}]}$ in the presence of piperidine, a catalytic amount 5 of copper iodide and $\mathrm{Pd}\left(\mathrm{PPh}_{3}\right)_{4}$ catalyst provided an excellent yield of the cross-coupling products $\mathbf{2 4}$. The selective reduction of enynes $\mathbf{2 4}$ by catalytic hydrogenation using Rosemund's catalyst gave saturated side chain derivatives 25 in excellent yield. Alternatively, alkyl compounds 25 were synthesized by $B$ 10 alkyl Suzuki cross-coupling between triflate $\mathbf{1 2}^{[2 \mathrm{c}]}$ and alkyl boranes 23 using $\mathrm{Pd}\left(\mathrm{PPh}_{3}\right)_{4}$ as catalyst and in the presence of $\mathrm{K}_{3} \mathrm{PO}_{4}$. Alkyl boranes 23 were prepared by reaction of the Grignard derivative of $\mathbf{8}$ with allyl bromide followed by hydroboration with $9-\mathrm{BBN}-\mathrm{H}$ of the corresponding allyl 15 derivative 22. Finally, compounds $\mathbf{2 5}$ were converted to the desired acids $\mathbf{7}$ in the same way as acids $\mathbf{5}$ from lactones $\mathbf{1 3}$.

\section{Inhibition Assay Results}

The inhibitory properties of compounds 5-7 against DHQ2-Hp 20 and DHQ2-Mt were tested. These compounds proved to be reversible competitive inhibitors of both enzymes. The inhibition data $\left(K_{\mathrm{i}}\right)$ are summarised in Table 4.

\begin{tabular}{|c|c|c|c|c|}
\hline Entry & Comp & $\mathrm{R}$ & H. pylori ${ }^{[\mathrm{a}]}$ & M. tuberculosis ${ }^{[b]}$ \\
\hline 1 & $5 \mathbf{a}$ & $(E) \mathrm{CH}=\mathrm{CH}$ & $1400 \pm 98$ & $780 \pm 94$ \\
\hline 2 & $5 b$ & $(E) \mathrm{CH}=\mathrm{CH}$ & $3110 \pm 249$ & $520 \pm 31$ \\
\hline 3 & $6 a$ & $\left(\mathrm{CH}_{2}\right)_{2}$ & $790 \pm 29$ & $436 \pm 13$ \\
\hline 4 & $6 b$ & $\left(\mathrm{CH}_{2}\right)_{2}$ & $2460 \pm 197$ & $254 \pm 20$ \\
\hline 5 & $6 c$ & $\left(\mathrm{CH}_{2}\right)_{2}$ & $1150 \pm 115$ & $274 \pm 16$ \\
\hline 6 & $7 a$ & $\left(\mathrm{CH}_{2}\right)_{3}$ & $243 \pm 19$ & $180 \pm 9$ \\
\hline 7 & $7 d$ & $\left(\mathrm{CH}_{2}\right)_{3}$ & $295 \pm 10$ & $73 \pm 4$ \\
\hline 8 & $4 a$ & $\mathrm{OCH}_{2}$ & $310 \pm 46^{[5 b]}$ & $35 \pm 2^{[5 b]}$ \\
\hline 9 & $4 b$ & $\mathrm{OCH}_{2}$ & $132 \pm 13^{[5 \mathrm{a}]}$ & $28 \pm 2^{[5 b]}$ \\
\hline
\end{tabular}

${ }_{25}$ The biological results show that, in general, the effects of type, geometry and size of spacer were more pronounced in the inhibition potency against the DHQ2-Hp enzyme and in all cases the propylene spacer was the most potent of the series for both enzymes. In general, compounds $\mathbf{6}$ and $\mathbf{7}$ having a flexible spacer 30 proved to be more potent than compounds $\mathbf{5}$ with a more rigid one (Table 4, entry 6 vs 1). Benzothiophene $7 \mathbf{d}$ having a propylene spacer was the most potent compound in the series, with $K_{\mathrm{i}}$ values of $73 \mathrm{nM}$ and $295 \mathrm{nM}$ against DHQ2-Mt and DHQ2-Hp, respectively. Naphthyl derivative 7a also showed a 35 high affinity against both enzymes, with $K_{\mathrm{i}}$ values of $180 \mathrm{nM}$ and $243 \mathrm{nM}$ against DHQ2-Mt and DHQ2-Hp, respectively. In addition, tetrahydronaphathalene $\mathbf{6 c}$ proved to have binding affinities against DHQ2 in the same range as the other unsaturated analogs $\mathbf{6 a}-\mathbf{b}$ (Table 4, entry 5 vs 3). In order to get
40 an insight of the binding mode of these inhibitors, docking studies using GOLD 5.0.1 ${ }^{[9]}$ were carried out, which are discussed below.

\section{Docking studies}

${ }_{45}$ The binding modes of inhibitors 5-7 with DHQ2 enzymes were studied using GOLD 5.0.1 ${ }^{[9]}$ with the enzyme geometries found in crystals of DHQ2-Hp and DHQ2-Mt binding to 3-methoxyaryl derivative 4c (PDB code: $2 \mathrm{WKS}^{[5 \mathrm{a}]}$ and $2 \mathrm{Y} 71,{ }^{[5 \mathrm{~b}]}$ respectively).

In general, 3-alkylaryl enol mimics with a three-carbon-atom 50 spacer, as in ligands $\mathbf{7}$, fit more efficiently into the active site than the corresponding ethylene ones (ligands 6) because they locate the aromatic ring closer to the aliphatic residues of the enzyme active site (leucine pocket). This fact may account for the higher inhibition potency of propylene derivatives 7 relative to inhibitors

55 and 6. The GOLD-predicted binding mode of one of the most active ligands of the 3-alkylaryl series, compound $\mathbf{7 d}$, in the active site of both enzymes is shown in Figure 4. These docking studies show that this inhibitor should have similar polar interactions, through hydroxyl and carboxylate groups (not 60 shown), to other mimetics of the enol intermediate, such as the ones present in the previously reported crystal structures (PDB code: $2 \mathrm{WKS}^{[5 \mathrm{a}]}$ and $2 \mathrm{Y} 1^{[5 \mathrm{~b}]}$ ), because the cyclohexene ring occupies approximately the same position in the active site. More importantly, in both cases, the benzothiophene ring and the 65 spacer are involved in a set of strong lipophilic interactions in this part of the active site. The benzothiophene moiety interacts with the essential tyrosine by $\pi$ stacking in DHQ2-Hp (Tyr22, Figure $4 \mathrm{a}$ ) and by an edge-face $\pi-\pi$ interaction in DHQ2-Mt (Tyr24, Figure $4 \mathrm{~b}$ ). This aromatic ring is also in close contact with the 70 side chain of Leu14 and the five-membered ring of Pro19 in DHQ2-Hp and the side chain of Leu16, the carbon side chain of Arg15 and the essential Arg19 in DHQ2-Mt. The latter residues are located in the flexible loop that closes over the substrate binding site. The benzothiophene ring also interacts with some 75 residues of a symmetry-related neighboring molecule (specifically, the side chains of Leu93, Met92 and Asp89 for DHQ2-Hp and the side chains of Ala91, Glu92 and Asp88 for DHQ2-Mt). The propylene moiety of 7d interacts with the side chain of Leu11/Leu14, the carbon side chain of Asn10/Asn12 and 80 carbon main chain of Gly78/Gly77 for DHQ2-Hp and DHQ2-Mt, respectively.

Comparison of saturated ligands $\mathbf{6}$ and the unsaturated ones 5 reveals that the saturated ones are predicted to be far more active than the corresponding unsaturated derivatives $\mathbf{5}$, because the 85 chain flexibility allows it to accommodate more adequately the aromatic ring in the active site, thus maximizing interactions (Figure 5). In fact, the GOLD-predicted binding mode of ligand 5a shows that the cyclohexene moiety is moved away from the polar contacts of the active site that anchors the six-membered 90 ring of the substrate and the enol intermediate in the active site, i.e. His82, His101, etc. (Figure 5). Even assuming that in a dynamic process the loop conformation and/or side chain residues might change, the ethylene spacer seems more suitable to maintain the polar interactions that anchor the cyclohexene 95 moiety of the inhibitor. 

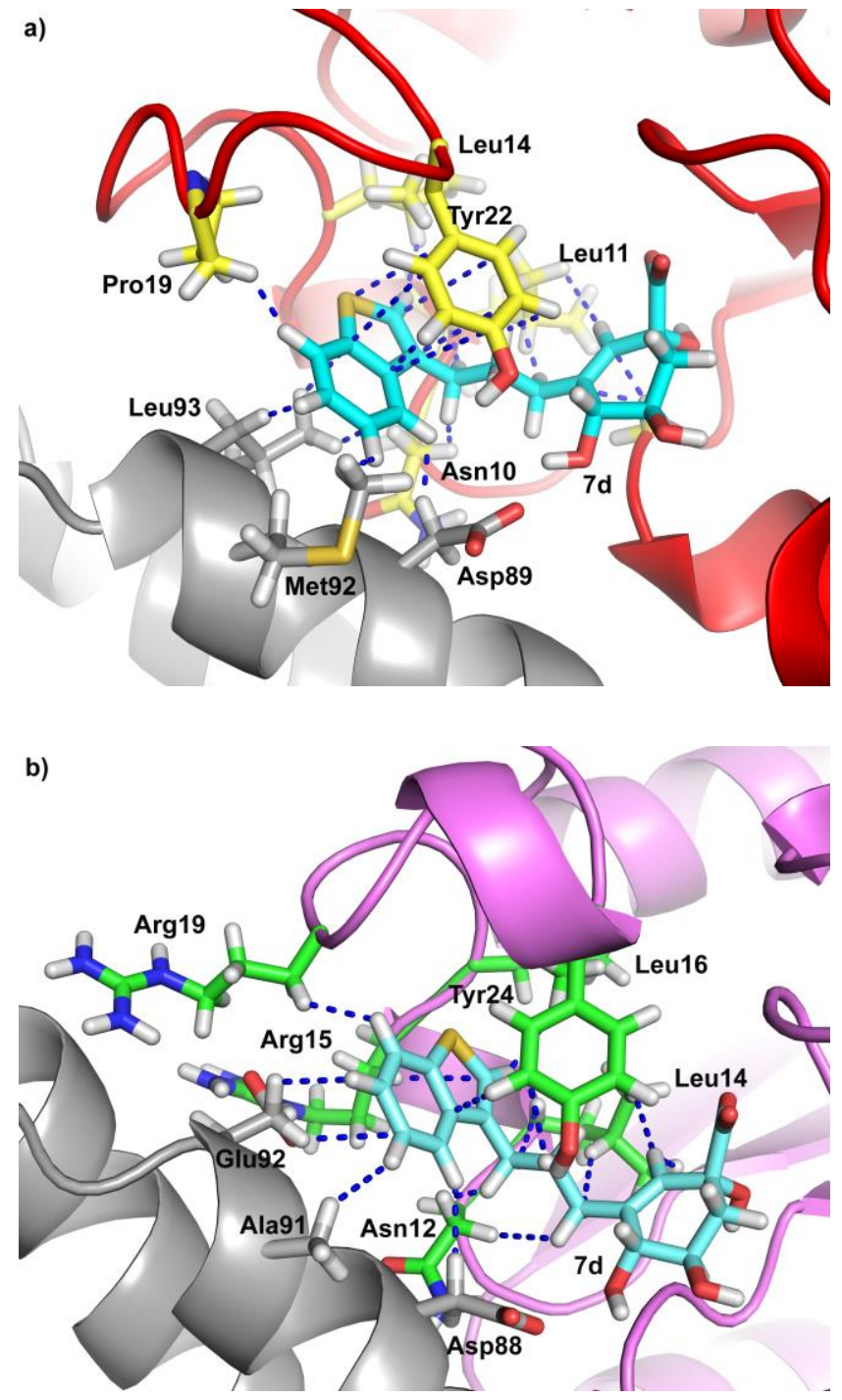

Figure 4. GOLD-predicted binding for ligand 7d to the active site of: a) DHQ2-Hp 5 (PDB: $2 \mathrm{WKS}^{[5 \mathrm{a}]}$ ); b) DHQ2-Mt (PDB: $2 \mathrm{Y} 1^{[5 \mathrm{~b}]}$ ). Relevant residues are indicated. Symmetry-related neighboring chain close to the active site is indicated in gray.

\section{Molecular Dynamics Simulations}

On the other hand, the inhibition data clearly show that the 10 replacement of the oxygen atom of the methylenoxy spacer by a carbon atom affords less potent inhibitors. This fact suggests that the oxygen atom of the spacer in compounds $\mathbf{4}$ is involved in a strong binding interaction with the essential water involved in the enzymatic mechanism, as described below. As shown in the 15 recently solved crystal structure of the binary complex DHQ2$\mathrm{Mt} / \mathbf{4 c}$, the oxygen atom of the spacer is located 3.1 $\mathrm{A}$ away from the essential water molecule (Figure 2b). Therefore, this interaction should be lost on replacing the ether linkage by a methylene group. In order to corroborate this hypothesis and

20 further analyze the binding mode of these inhibitors in the active site of the DHQ2, we studied the binding mode of $O$-alkylaryl derivative $\mathbf{4 c}$ and the corresponding $C$-alkylaryl derivative $\mathbf{6 e}(\mathrm{Ar}$ = 5-methylbenzo $[b]$ thiophen-3-yl) by molecular dynamics simulations (MD). The results show that the position in the active 25 site of 3-methoxybenzothiophenyl derivatives $\mathbf{4 c}$, which has a methylenoxy spacer, does not change significantly during the simulation $(10 \mathrm{~ns})$ - including its position relative to the catalytic water (Figures 6a-b).

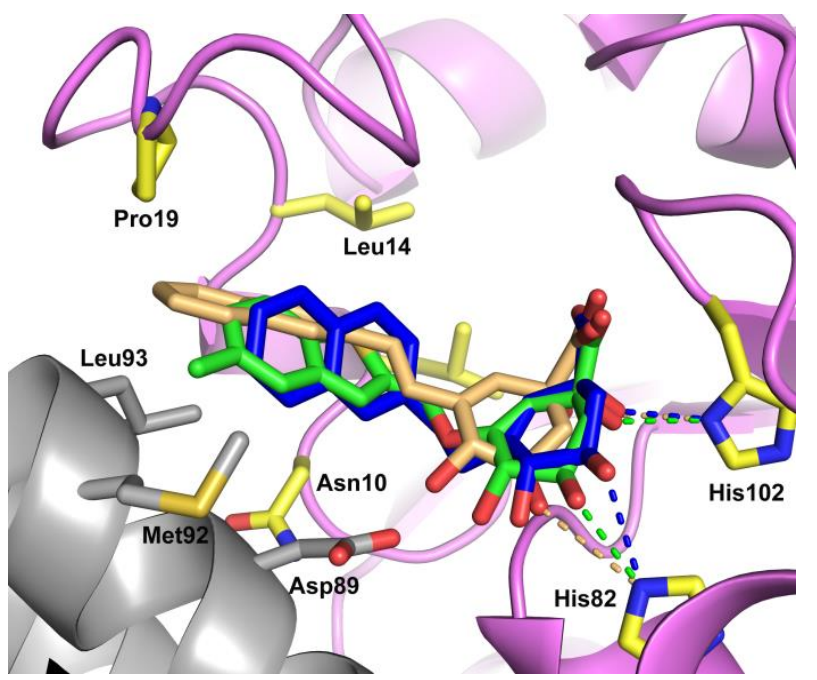

30 Figure 5. Comparison of the position of inhibitor $\mathbf{4 c}$ (green) in the enzyme-inhibitor crystal structure of DHQ2-Hp (PDB code: $2 \mathrm{WKS}^{[5 \mathrm{a}]}$ ) with the docking results of the highest score solution of ligands: 5a (pale orange) and 6a (blue). Relevant residues are indicated. The hydrogen bonding interactions of hydroxyl groups on $\mathrm{C}-1$ and $\mathrm{C}-5$ with His 82 and His 102 are highlighted as dotted lines with the same color as the 35 corresponding ligand. Note how these contacts are much weaker for ligand $\mathbf{5 a}$ than for compounds $\mathbf{6 a}$ and $\mathbf{4 c}$

For 3-ethylbenzothiophenyl ligand $\mathbf{6 e}$, which contains an ethylene spacer, relevant changes were not found in the position 40 of the cyclohexene moiety and therefore its polar contacts through hydroxyl and carboxylate groups with residues of the active site [His80, Arg111, Ser102, Asn74, His100, Asp89 (neighboring unit)]. However, an important change in the position and conformation of the side chain and the aromatic ring was 45 observed. Both moieties are shifted significantly after the simulation, which causes a change in the position of the loop because the volume occupied by the ligand $\mathbf{6 e}$ is now greater. As shown in Figure 6, while the distance between the oxygen atom of the methylenoxy spacer in ligand $\mathbf{4 c}$ does not change 50 significantly after $10 \mathrm{~ns}$ of simulation (from $3.1 \AA$ to $2.8 \AA$ ), the corresponding distance for ligand 6 e increases from $3.2 \AA$ to 4.2 $\AA$ (see also supporting information). Therefore, the substitution of the methylenoxy spacer by an alkylene one might cause the loss of a favorable polar interaction between the ligand and the 55 catalytic water and this in turn causes a loss of inhibition potency.

\section{Conclusions}

Several 3-alkylaryl mimics of the enol intermediate in the reaction catalyzed by the third enzyme of the shikimic acid pathway, i.e. type II dehydroquinase - an essential enzyme in $M$. 60 tuberculosis and $H$. pylori, were synthesized and tested as inhibitors of these enzymes. Vinylene derivatives 5 were synthesized by Suzuki cross-coupling reactions between previously reported triflate $12^{[2 c]}$ and boronic acids pinacol esters 11 as key step. 2- and 3-alkylaryl enol mimics 6 and 7 were 65 synthesized by $B$-alkyl Suzuki cross-coupling reactions using alkyl boranes 18 and 23, respectively. Ethylene $\mathbf{6}$ and propylene side-chain acids 7 were also synthesized by selective catalytic 
hydrogenation using Rosemund's catalyst or Raney-Ni of the external double and tripe bond in dienes $\mathbf{1 3}$ and enynes 24, respectively, which were obtained by Suzuki and Sonogashira cross-coupling reactions.

5 The reported compounds were synthesized to evaluate the contribution in the high potency of inhibitors $\mathbf{4}$ of the hydrogenbonding interaction between the oxygen atom of the methylenoxy spacer and the essential water involved in the catalysis, as well as the length and the rigidity of the alkylene spacer. The biological 10 results show that the replacement of the oxygen atom of the methylenoxy spacer of previously reported inhibitors $4 \mathbf{a}^{[5 a]}$ and $\mathbf{4} \mathbf{c}^{[5 b]}$ by a carbon atom leads to a decrease in the inhibition potency upto 20 -fold. The inhibition data toghether with the

molecular dynamics simulation studies performed show that this 15 hydrogen-bonding interaction has an important contribution on the inhibition potency of inhibitors $\mathbf{4}$ and it should therefore be considered in future designs. In general, effects of geometry and size of the alkyl spacer were more pronounced in the inhibition potency against the DHQ2-Hp enzyme and in all cases 20 compounds $\mathbf{6}$ and $\mathbf{7}$ having a flexible spacer proved to be more potent than compounds $\mathbf{5}$ with a more rigid one. Docking studies using the program GOLD 5.0.1 suggest that compounds with a three-carbon spacer fit more efficiently into the active site because they locate the aromatic ring closer to the aliphatic ${ }_{25}$ residues of the enzyme active site.

b)
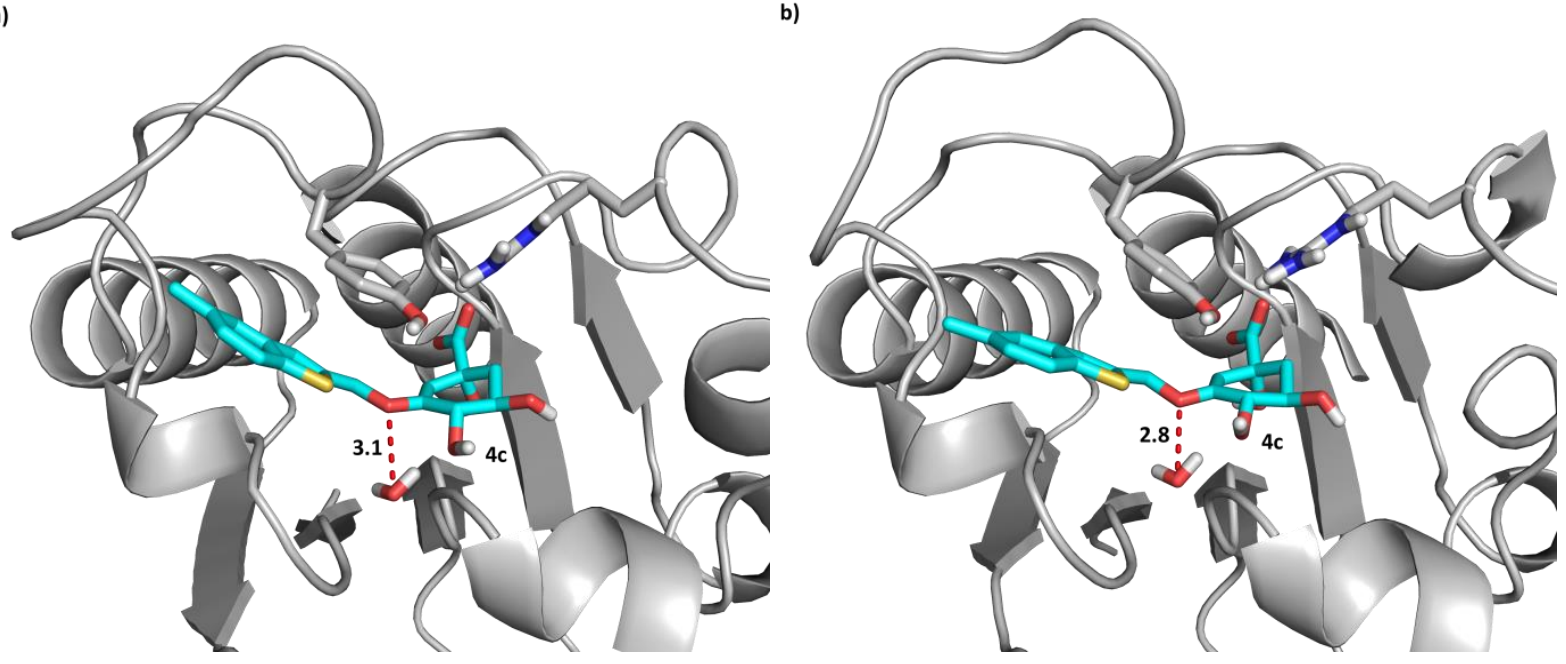

c)

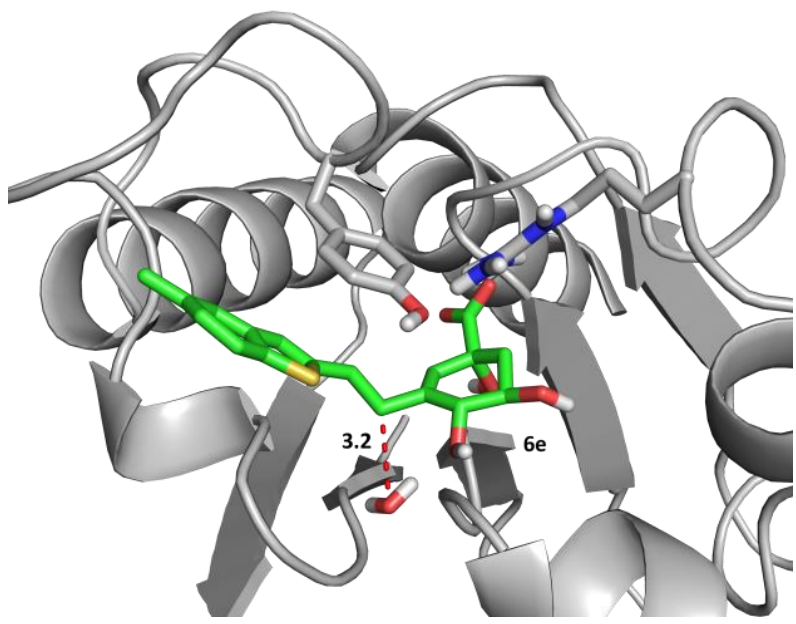

d)

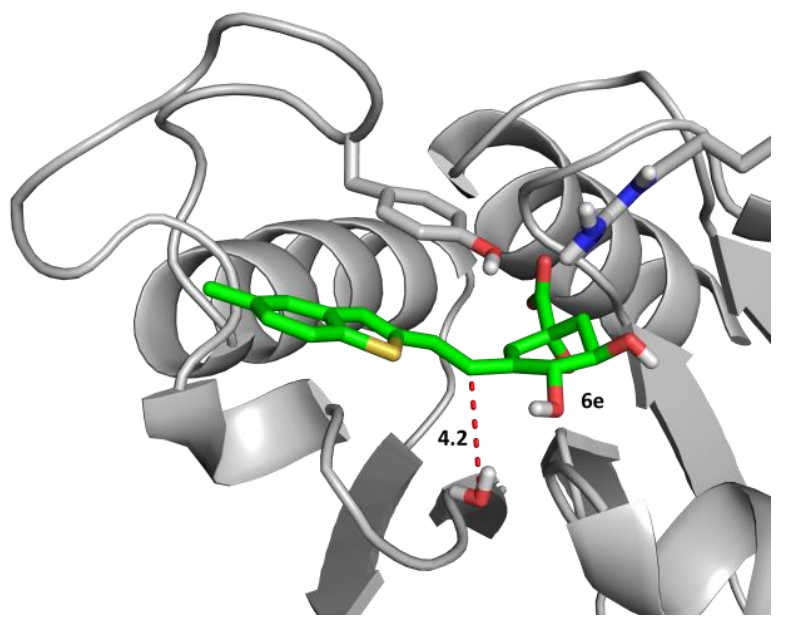

Figure 6. Binding mode of ligand $\mathbf{4 c}$ (cyan) and ligand 6e (green) in the active site of DHQ2-Mt obtained by MD simulations: a and c) after minimization and previously to 30 simulation; b and d) after $10 \mathrm{~ns}$ of MD. Distance between the oxygen atom of the spacer of ligand $\mathbf{4 c}$ (O6), the corresponding carbon atom in ligand $\mathbf{6 e}$ (C8) and essential water molecule is indicated. Only relevant residues are indicated.

\section{Experimental}

\section{General}

All starting materials and reagents were commercially available 35 and were used without further purification. ${ }^{1} \mathrm{H}$ NMR spectra (250, 300,400 and $500 \mathrm{MHz}$ ) and ${ }^{13} \mathrm{C}$ NMR spectra $(63,75,100$ and $125 \mathrm{MHz}$ ) were measured in deuterated solvents. $J$ values are given in Hertz. NMR assignments were carried out by a combination of $1 \mathrm{D}, \mathrm{COSY}$, and DEPT-135 experiments. FT-IR 40 spectra were recorded as $\mathrm{NaCl}$ plates or $\mathrm{KBr}$ discs. $[\alpha]_{20}^{\mathrm{D}}=$ values are given in $10^{-1} \mathrm{deg} \mathrm{cm}^{2} \mathrm{~g}^{-1}$. All procedures involving the use of ion-exchange resins were carried out at room temperature using Milli-Q deionized water. Amberlite IR-120 ( $\left.\mathrm{H}^{+}\right)$(cation exchanger) was washed alternately with water, $10 \% \mathrm{NaOH}$, 
water, $10 \% \mathrm{HCl}$, and finally water before use. HPLC was performed on a semipreparative column (Phenomenex Luna, 250 $\times 21.2 \mathrm{~mm}, \mathrm{C} 18$ ), eluting with acetonitrile-water at a flow rate of $7 \mathrm{~mL} \mathrm{~min}^{-1}$.

Trimethyl(3-(naphthalen-2-yl)ethynyl)silane (9a)

A Shlenck tube was charged with 2-bromonaphthalene (8a) (500 $\mathrm{mg}, 2.41 \mathrm{mmol}), \mathrm{Pd}\left(\mathrm{PPh}_{3}\right)_{2} \mathrm{Cl}_{2}$ (105 mg, $\left.0.14 \mathrm{mmol}\right), \mathrm{CuI}$ (25 mg, $0.14 \mathrm{mmol})$ and dry triethylamine $(5 \mathrm{~mL})$. The resulting solution 10 was deoxygenated and ethynyltrimethylsilane $(0.5 \mathrm{~mL}, 3.62$ mmol) was added dropwise. After addition of the first drop, the reaction color changed from yellow to black. The resulting solution was heated at $40{ }^{\circ} \mathrm{C}$ for $5 \mathrm{~h}$. After cooling to room temperature, saturated ammonium chloride $(0.5 \mathrm{~mL})$ was added 15 and the reaction mixture was extracted with diethyl ether $(\times 3)$. The combined organic extracts were dried ( $\left.a n h . \mathrm{Na}_{2} \mathrm{SO}_{4}\right)$, filtered and concentrated under reduced pressure. The residue obtained was purified by flash chromatography on silica gel, eluting with hexanes to give silane $9 \mathbf{a}(534 \mathrm{mg}, 99 \%)$ as a brown oil. $\delta_{\mathrm{H}}(250$ $\left.20 \mathrm{MHz} ; \mathrm{CDCl}_{3}\right): 8.19(1 \mathrm{H}$, br s, $\mathrm{ArH}), 7.90-7.83(3 \mathrm{H}, \mathrm{m}, 3 \times \mathrm{ArH})$, $7.70(1 \mathrm{H}, \mathrm{dd}, J=7.5$ and $1.5 \mathrm{~Hz}, \operatorname{ArH}), 7.56(2 \mathrm{H}, \mathrm{dd}, J=6.3$ and $3.2 \mathrm{~Hz}, 2 \times \mathrm{ArH})$ and $0.52\left(9 \mathrm{H}, \mathrm{s}, 3 \times \mathrm{SiCH}_{3}\right) ; \delta_{\mathrm{C}}(63 \mathrm{MHz}$; $\left.\mathrm{CDCl}_{3}\right)$ : $133.0(2 \times \mathrm{C}), 132.1(\mathrm{CH}), 128.6(\mathrm{CH}), 128.0(2 \times \mathrm{CH})$, $127.8(\mathrm{CH}), 126.8(\mathrm{CH}), 126.6(\mathrm{CH}), 120.5(\mathrm{C}), 106.8(\mathrm{C}), 95.6$ ${ }_{25}(\mathrm{C})$ and $0.2\left(3 \times \mathrm{SiCH}_{3}\right) ; \mathrm{v}_{\max }($ film $) / \mathrm{cm}^{-1} 2152(\mathrm{C} \equiv \mathrm{C})$.

\section{(Benzo[b]thiophen-2-ylethynyl)trimethylsilane (9b)}

The experimental procedure used was the same as for alkyne 9a utilizing 2-bromobenzo[b]thiophene (8b) (1 g, $4.69 \mathrm{mmol})$. 30 Yield $=1.05 \mathrm{~g}(98 \%)$. White solid. $\mathrm{Mp}: 57-58^{\circ} \mathrm{C} ; \delta_{\mathrm{H}}(250 \mathrm{MHz}$; $\left.\mathrm{CDCl}_{3}\right)$ : 7.81-7.75 $(2 \mathrm{H}, \mathrm{m}, 2 \times \mathrm{ArH}), 7.52(1 \mathrm{H}$, br s, $\mathrm{ArH})$, 7.41-7.37 $(2 \mathrm{H}, \mathrm{m}, 2 \times \mathrm{ArH})$ and $0.37\left(9 \mathrm{H}, \mathrm{s}, 3 \times \mathrm{SiCH}_{3}\right) ; \delta_{\mathrm{C}}(63$ $\left.\mathrm{MHz} ; \mathrm{CDCl}_{3}\right)$ : $140.2(\mathrm{C}), 139.0(\mathrm{C}), 129.6(\mathrm{CH}), 125.6(\mathrm{CH})$, $124.8(\mathrm{CH}), 124.0(\mathrm{CH}), 123.2(\mathrm{C}), 122.1(\mathrm{CH}), 101.1(\mathrm{C}), 98.0$ $35(\mathrm{C})$, and $0.1\left(3 \times \mathrm{SiCH}_{3}\right) ; \mathrm{v}_{\max }(\mathrm{KBr}) / \mathrm{cm}^{-1} 2143(\mathrm{C} \equiv \mathrm{C})$; MS (ESI) $\mathrm{m} / \mathrm{z} 231\left(\mathrm{MH}^{+}\right)$; HRMS (ESI) calcd for $\mathrm{C}_{13} \mathrm{H}_{15} \mathrm{SSi}\left(\mathrm{MH}^{+}\right)$: 231.0658 , found 231.0666 .

\section{2-Ethynylnaphthalene (10a)}

40 Tetrabutylammonium fluoride $(2.9 \mathrm{~mL}, 2.87 \mathrm{mmol}, \mathrm{ca} 1.0 \mathrm{M}$ in THF) was added to a stirred solution of the silyl ether 9a (534 $\mathrm{mg}, 2.39 \mathrm{mmol})$ in dry THF $(25 \mathrm{~mL})$ under argon at room temperature. After stirring for $1 \mathrm{~h}$ the solvent was removed under reduced pressure and the residue was dissolved in ethyl acetate 45 and $\mathrm{HCl}(10 \%)$. The organic layer was separated and the aqueous layer was extracted with ethyl acetate $(\times 2)$. The combined organic extracts were dried (anh. $\mathrm{Na}_{2} \mathrm{SO}_{4}$ ), filtered and concentrated under reduced pressure. The residue was purified by flash chromatography on silica gel, eluting with hexanes to yield 50 alkyne 10a (354 mg, 98\%) as a colorless oil. $\delta_{\mathrm{H}}(250 \mathrm{MHz}$; $\left.\mathrm{CDCl}_{3}\right): 8.19$ (1 H, s, ArH), 7.92-7.85 (3 H, m, 3×ArH), 7.71 (1 $\mathrm{H}$, dd, $J=8.5$ and $1.6 \mathrm{~Hz}, \mathrm{ArH}), 7.60-7.57(2 \mathrm{H}, \mathrm{m}, \mathrm{ArH})$ and $3.36(1 \mathrm{H}, \mathrm{s}, \mathrm{CH}) ; \delta_{\mathrm{H}}\left(63 \mathrm{MHz} ; \mathrm{CDCl}_{3}\right): 133.0(\mathrm{C}), 132.8(\mathrm{C})$, $132.3(\mathrm{CH}), 128.5(\mathrm{CH}), 128.0(\mathrm{CH}), 127.7(2 \times \mathrm{CH}), 126.9(\mathrm{CH})$, $55126.6(\mathrm{CH}), 119.4(\mathrm{C}), 84.1(\mathrm{C})$ and $77.7(\mathrm{CH}) ; v_{\max }(\mathrm{KBr}) / \mathrm{cm}^{-1}$ $2104(\mathrm{C} \equiv \mathrm{C})$.

\section{2-Ethynylbenzo $[b]$ thiophene $(10 \mathrm{~b})$}

The experimental procedure used was the same as for 260 ethynylnaphthalene (10a) utilizing silyl ether $9 b(1.05 \mathrm{~g}, 4.58$ mmol $)$. Yield $=630 \mathrm{mg}(87 \%)$. Red Liquid. $\delta_{\mathrm{H}}(250 \mathrm{MHz}$; $\left.\mathrm{CDCl}_{3}\right)$ : 7.84-7.78 (2 H, m, 2×ArH), 7.58 (1 H, s, ArH), 7.45-7.41 (2 H, m, 2×ArH) and $3.53(1 \mathrm{H}, \mathrm{s}, \mathrm{CH}) ; \delta_{\mathrm{C}}(63 \mathrm{MHz}$; $\left.\mathrm{CDCl}_{3}\right): 140.1(\mathrm{C}), 138.7(\mathrm{C}), 130.1(\mathrm{CH}), 125.8(\mathrm{CH}), 124.8$ $65(\mathrm{CH}), 124.0(\mathrm{CH}), 122.0(\mathrm{CH}), 121.9(\mathrm{C}), 83.2(\mathrm{C})$ and 77.3 $(\mathrm{CH}) ; v_{\max }(\mathrm{film}) / \mathrm{cm}^{-1} 2100(\mathrm{C} \equiv \mathrm{C})$.

\section{(E)-4,4,5,5-tetramethyl-2-(2-(naphth-2-yl)vinyl)-1,3,2- dioxaborolane (11a)}

${ }_{70}$ A Shlenck tube was charged with 2-ethynylnaphthalene (10a) (1.54 g, $10.14 \mathrm{mmol})$, catecholborane $(1.28 \mathrm{~mL}, 11.15 \mathrm{mmol})$ and dry THF $(2 \mathrm{~mL})$. The resultant solution was heated under reflux for $12 \mathrm{~h}$. After cooling to room temperature, a solution of pinacol (3.85 g, $32.57 \mathrm{mmol})$ in dry THF $(20 \mathrm{~mL})$ was added. The 75 reaction mixture was heated under reflux for $19 \mathrm{~h}$. After cooling to room temperature, the solvent was removed under reduced pressure. The residue was purified by flash chromatography on silica gel, preneutralized with (1:2:97) triethylamine/diethyl ether/hexanes, using (3:97) diethyl ether/hexanes as eluent, to 80 give boronic acid pinacol ester 11a $(2.43 \mathrm{~g}, 85 \%)$ as a yellow oil. $\delta_{\mathrm{H}}\left(250 \mathrm{MHz} ; \mathrm{CDCl}_{3}\right): 7.83(4 \mathrm{H}, \mathrm{m}, 4 \times \mathrm{ArH}), 7.61(1 \mathrm{H}, \mathrm{d}, J=$ $18.5 \mathrm{~Hz}, \mathrm{CH}=\mathrm{CHB}), 7.48$ (3 H, m, 3xArH), $6.33(1 \mathrm{H}, \mathrm{d}, J=18.5$ $\mathrm{Hz}, \mathrm{CH}=\mathrm{CHB}), 1.36\left(9 \mathrm{H}, \mathrm{s}, 3 \times \mathrm{CH}_{3}\right)$ and $1.24\left(3 \mathrm{H}, \mathrm{s}, \mathrm{CH}_{3}\right) ; \delta_{\mathrm{C}}$ $\left(63 \mathrm{MHz} ; \mathrm{CDCl}_{3}\right): 149.5(\mathrm{CH}), 134.9(\mathrm{C}), 133.7(\mathrm{C}), 133.4(\mathrm{C})$, $85128.4(\mathrm{CH}), 128.2(\mathrm{CH}), 128.0(\mathrm{CH}), 127.6(2 \times \mathrm{CH}), 126.4(\mathrm{CH})$, $126.2(\mathrm{CH}), 124.9(\mathrm{CH}), 123.3(\mathrm{CH}), 83.3(2 \times \mathrm{C})$ and 24.8 $\left(4 \times \mathrm{CH}_{3}\right)$.

\section{(E)-2-(2-(Benzo[b]thiophen-2-yl)vinyl)-4,4,5,5-tetramethyl- 90 1,3,2-dioxaborolane (11b)}

The experimental procedure used was the same as for dioxaborolane 11a utilizing alkyne 10b $(600 \mathrm{mg}, 3.79 \mathrm{mmol})$. Yield: $1.03 \mathrm{~g}(94 \%)$. Yellow oil. $\delta_{\mathrm{H}}\left(250 \mathrm{MHz} ; \mathrm{CDCl}_{3}\right)$ : 7.84-7.76 (2 H, m, 2×ArH), $7.69(1 \mathrm{H}, \mathrm{d}, J=18.0 \mathrm{~Hz}$, $\left.{ }_{95} \mathrm{CH}=\mathrm{CHB}\right), 7.35-7.32(2 \mathrm{H}, \mathrm{m}, 2 \times \mathrm{ArH}), 7.29(1 \mathrm{H}, \mathrm{s}, \mathrm{ArH}), 6.16$ $(1 \mathrm{H}, \mathrm{d}, J=18.0 \mathrm{~Hz}, \mathrm{CH}=\mathrm{CHB})$ and $1.38\left(12 \mathrm{H}, \mathrm{s}, 4 \times \mathrm{CH}_{3}\right) ; \delta_{\mathrm{C}}$ (63 MHz; $\left.\mathrm{CDCl}_{3}\right): 143.8(\mathrm{C}), 142.3(\mathrm{CH}), 139.8(\mathrm{C}), 139.5(\mathrm{C})$, $125.2(\mathrm{CH}), 125.0(\mathrm{CH}), 124.4(\mathrm{CH}), 123.9(2 \times \mathrm{CH}), 122.2(\mathrm{CH})$, $83.3(2 \times \mathrm{C})$ and $24.7\left(4 \times \mathrm{CH}_{3}\right)$.

100

$(1 R, 4 R, 5 R)-1,4-D i(t e r t-b u t y l d i m e t h y l s i l y l o x y)-3-((E)-2-$ (naphth-2-yl)vinyl)cyclohex-2-en-1,5-carbolactone (13a)

A Shlenck tube was charged with triflate $\mathbf{1 2}^{[2 \mathrm{c}]}(57 \mathrm{mg}, 0.11$ $\mathrm{mmol}), \mathrm{Pd}\left(\mathrm{PPh}_{3}\right)_{4}(4.1 \mathrm{mg}, 0.035 \mathrm{mmol})$ and dry dioxane $(1 \mathrm{~mL})$. $105 \mathrm{~K}_{3} \mathrm{PO}_{4}(0.18 \mathrm{~mL}, 0.18 \mathrm{mmol}, 1 \mathrm{M})$ and dioxaborolane 11a $(60$ $\mathrm{mg}, \quad 0.21 \mathrm{mmol})$ were added. The resultant solution was deoxygenated and heated at $80{ }^{\circ} \mathrm{C}$ for $3.5 \mathrm{~h}$ under argon. After cooling to room temperature, the solvent was removed under reduced pressure. The residue was dissolved in a mixture of 110 dichloromethane and water. The organic layer was separated and the aqueous phase was extracted with dichloromethane $(\times 2)$. The combined organic extracts were dried $\left(\mathrm{Na}_{2} \mathrm{SO}_{4}\right)$, filtered and concentrated under reduced pressure. The residue obtained was purified by flash chromatography on silica gel, eluting with a 115 gradient of dichloromethane-hexanes (15:85 to $25: 75)$, to give naphthyl derivative 13a (56 mg, 94\%) as a white foam. $[\alpha]_{20}^{\mathrm{D}}=-$ 
$9.8^{\circ}\left(c 1.0\right.$ in $\left.\mathrm{CHCl}_{3}\right) ; \delta_{\mathrm{H}}\left(250 \mathrm{MHz} ; \mathrm{CDCl}_{3}\right): 7.85-7.74(4 \mathrm{H}, \mathrm{m}$, 4×ArH), 7.50-7.41 (3 H, m, 3×ArH), $6.89(1 \mathrm{H}, \mathrm{d}, J=16.3 \mathrm{~Hz}$, $\mathrm{ArCH}=\mathrm{CH}), 6.70(1 \mathrm{H}, \mathrm{d}, J=16.3 \mathrm{~Hz}, \mathrm{ArCH}=\mathrm{CH}), 6.18(1 \mathrm{H}, \mathrm{s}$, $\mathrm{H}-2), 4.66$ (1 H, m, H-5), 4.58 (1 H, d, J=3.2 Hz, H-4), 2.55 (1 $\left.{ }_{5} \mathrm{H}, \mathrm{d}, J=10.6 \mathrm{~Hz}, \mathrm{H}-6_{\mathrm{ax}}\right), 2.41\left(1 \mathrm{H}, \mathrm{m}, \mathrm{H}-6_{\mathrm{eq}}\right), 1.00(9 \mathrm{H}, \mathrm{s}$, $\left.\mathrm{C}\left(\mathrm{CH}_{3}\right)_{3}\right), 0.94\left(9 \mathrm{H}, \mathrm{s}, \mathrm{C}\left(\mathrm{CH}_{3}\right)_{3}\right), 0.30\left(3 \mathrm{H}, \mathrm{s}, \mathrm{SiCH}_{3}\right), 0.28(3 \mathrm{H}$, $\left.\mathrm{s}, \mathrm{SiCH}_{3}\right), 0.26\left(3 \mathrm{H}, \mathrm{s}, \mathrm{SiCH}_{3}\right)$ and $0.24\left(3 \mathrm{H}, \mathrm{s}, \mathrm{SiCH}_{3}\right) ; \delta_{\mathrm{C}}(63$ $\left.\mathrm{MHz} ; \mathrm{CDCl}_{3}\right): 175.4(\mathrm{C}), 136.7$ (C), $134.1(\mathrm{C}), 134.1(\mathrm{CH}), 133.7$ (C), $133.2(\mathrm{C}), 130.6(\mathrm{CH}), 128.5(\mathrm{CH}), 128.1(\mathrm{CH}), 127.8(\mathrm{CH})$, $10126.8(2 \times \mathrm{CH}), 126.5(\mathrm{CH}), 126.2(\mathrm{CH}), 123.2(\mathrm{CH}), 83.2(\mathrm{C})$, $75.3(\mathrm{CH}), 66.2(\mathrm{CH}), 37.2\left(\mathrm{CH}_{2}\right), 25.7\left(2 \times \mathrm{C}\left(\mathrm{CH}_{3}\right)_{3}\right), 18.1$ $\left(2 \times C\left(\mathrm{CH}_{3}\right)_{3}\right),-2.9\left(2 \times \mathrm{CH}_{3}\right),-3.9\left(\mathrm{CH}_{3}\right)$ and $-4.1\left(\mathrm{CH}_{3}\right) ; \mathrm{v}_{\max }$ (film) $/ \mathrm{cm}^{-1} 1799$ (CO) $\mathrm{cm}^{-1}$; MS (ESI) $\mathrm{m} / z=559\left(\mathrm{MNa}^{+}\right)$; HRMS (ESI) calcd for $\mathrm{C}_{31} \mathrm{H}_{44} \mathrm{O}_{4} \mathrm{SiNa}\left(\mathrm{MNa}^{+}\right)$: 559.2670, found 15559.2670 .

$(1 R, 4 R, 5 R)-3-((E)-2-(b e n z o[b]$ thiophen-2-yl)vinyl)-1,4-di $($ tertbutyl-dimethylsilyloxy)cyclohex-2-en-1,5-carbolactone (13b)

The experimental procedure used was the same as for compound 20 13a utilizing triflate $\mathbf{1 2}^{[2 \mathrm{c}]}(355 \mathrm{mg}, \quad 0.67 \mathrm{mmol})$ and dioxaborolane 11b $(382 \mathrm{mg}, 1.34 \mathrm{mmol})$. White foam. Yield = $315 \mathrm{mg}(87 \%) \cdot[\alpha]^{\mathrm{D}}{ }_{20}=-94.4^{\circ}\left(c 1.0\right.$ in $\left.\mathrm{CHCl}_{3}\right) ; \delta_{\mathrm{H}}(250 \mathrm{MHz}$; $\left.\mathrm{CDCl}_{3}\right)$ : 7.86-7.76 (2 H, m, 2×ArH), 7.43-7.36 (2 H, m, 2×ArH), $7.26(1 \mathrm{H}, \mathrm{s}, \mathrm{ArH}), 7.03(1 \mathrm{H}, \mathrm{d}, J=16.0 \mathrm{~Hz}, \mathrm{ArCH}=\mathrm{CH}), 6.54$ (1 $\left.{ }_{25} \mathrm{H}, \mathrm{d}, J=16.0 \mathrm{~Hz}, \mathrm{ArCH}=\mathrm{CH}\right), 6.26(1 \mathrm{H}, \mathrm{s}, \mathrm{H}-2), 4.73(1 \mathrm{H}, \mathrm{dd}, J$ $=3.3$ and $5.2 \mathrm{~Hz}, \mathrm{H}-5), 4.58(1 \mathrm{H}, \mathrm{d}, J=3.3 \mathrm{~Hz}, \mathrm{H}-4), 2.60(1 \mathrm{H}$, $\left.\mathrm{d}, J=10.6 \mathrm{~Hz}, \mathrm{H}-6_{\mathrm{ax}}\right), 2.55-2.42\left(1 \mathrm{H}, \mathrm{m}, \mathrm{H}-6_{\mathrm{eq}}\right), 1.08(9 \mathrm{H}, \mathrm{s}$, $\left.\mathrm{C}\left(\mathrm{CH}_{3}\right)_{3}\right), 1.03\left(9 \mathrm{H}, \mathrm{s}, \mathrm{C}\left(\mathrm{CH}_{3}\right)_{3}\right), 0.38\left(3 \mathrm{H}, \mathrm{s}, \mathrm{CH}_{3}\right), 0.36(3 \mathrm{H}, \mathrm{s}$, $\left.\mathrm{CH}_{3}\right), 0.33\left(3 \mathrm{H}, \mathrm{s}, \mathrm{CH}_{3}\right)$ and $0.31\left(3 \mathrm{H}, \mathrm{s}, \mathrm{CH}_{3}\right) ; \delta_{\mathrm{C}}(63 \mathrm{MHz}$; ${ }_{30} \mathrm{CDCl}_{3}$ ): $175.2(\mathrm{C}), 142.2$ (C), 140.1 (C), 139.1 (C), 136.0 (C), $134.5(\mathrm{CH}), 128.4(\mathrm{CH}), 125.0(\mathrm{CH}), 124.6(\mathrm{C}), 124.4(\mathrm{CH})$, $123.8(\mathrm{CH}), 123.6(\mathrm{CH}), 122.3(\mathrm{CH}), 75.8(\mathrm{CH}), 75.2(\mathrm{C}), 66.3$ $(\mathrm{CH}), 37.1\left(\mathrm{CH}_{2}\right), 25.7\left(2 \times \mathrm{C}\left(\mathrm{CH}_{3}\right)_{3}\right), 18.1\left(2 \times C\left(\mathrm{CH}_{3}\right)_{3}\right),-2.9$ $\left(2 \times \mathrm{CH}_{3}\right),-3.9\left(\mathrm{CH}_{3}\right)$ and $-4.3\left(\mathrm{CH}_{3}\right) ; \mathrm{v}_{\max }($ film $) / \mathrm{cm}^{-1} 1799$ 35 (CO); MS (ESI) $\mathrm{m} / \mathrm{z}=543\left(\mathrm{MH}^{+}\right)$; HRMS (ESI) calcd for $\mathrm{C}_{29} \mathrm{H}_{43} \mathrm{O}_{4} \mathrm{SSi}_{2}\left(\mathrm{MH}^{+}\right)$: 543.2415, found 543.2404.

$(1 R, 4 R, 5 R)-1,4-D i h y d r o x y-3-((E)-2-($ naphth-2yl)vinyl)cyclohex-2-en-1,5-carbolactone (14a)

40 The experimental procedure used was the same as for alkyne 9a utilizing silyl ether 13a $(290 \mathrm{mg}, 0.54 \mathrm{mmol})$. Purification by flash chromatography over silica gel, eluting with (1:1) ethyl acetate/hexanes gave diol 14a (107 mg, 65\%) as a colorless oil. $[\alpha]_{20}^{\mathrm{D}_{20}}=-95.7^{\circ}(c 1.0$ in $\mathrm{MeOH}) ; \delta_{\mathrm{H}}\left(250 \mathrm{MHz} ; \mathrm{CD}_{3} \mathrm{OD}\right): 7.80$ ${ }_{45} 7.75(4 \mathrm{H}, \mathrm{m}, 4 \times \mathrm{ArH}), 7.64(1 \mathrm{H}, \mathrm{dd}, J=8.7$ and $1.3 \mathrm{~Hz}, \mathrm{ArH})$, 7.44-7.39 (2 H, m, 2×ArH), $7.11(1 \mathrm{H}, \mathrm{d}, J=16.4 \mathrm{~Hz}$, $\mathrm{ArCH}=\mathrm{CH}), 6.82(1 \mathrm{H}, \mathrm{d}, J=16.4 \mathrm{~Hz}, \mathrm{ArCH}=\mathrm{CH}), 6.16(1 \mathrm{H}, \mathrm{s}$, $\mathrm{H}-2), 4.73$ (1 H, m, H-5), $4.54(1 \mathrm{H}, \mathrm{d}, J=3.2 \mathrm{~Hz}, \mathrm{H}-4)$ and 2.49-2.41 (2 H, m, $\left.\mathrm{CH}_{2}\right) ; \delta_{\mathrm{C}}\left(63 \mathrm{MHz} ; \mathrm{CD}_{3} \mathrm{OD}\right): 178.4(\mathrm{C}), 138.4$ $50(\mathrm{C}), 135.8(\mathrm{C}), 135.1(\mathrm{C}), 134.8(\mathrm{CH}), 134.6(\mathrm{C}), 132.2(\mathrm{CH})$, $129.3(\mathrm{CH}), 129.0(\mathrm{CH}), 128.6(\mathrm{CH}), 128.3(\mathrm{CH}), 127.8(\mathrm{CH})$, $127.4(\mathrm{CH}), 127.1(\mathrm{CH}), 124.3(\mathrm{CH}), 78.0(\mathrm{CH}), 74.5(\mathrm{C}), 65.8$ $\left(\mathrm{CH}_{2}\right)$ and $37.6\left(\mathrm{CH}_{2}\right) ; v_{\max }($ film $) / \mathrm{cm}^{-1} 3381(\mathrm{OH})$ and 1772 (CO); MS (ESI) $\mathrm{m} / \mathrm{z}=331\left(\mathrm{MNa}^{+}\right)$; HRMS (ESI) calcd for ${ }_{55} \mathrm{C}_{19} \mathrm{H}_{16} \mathrm{O}_{4} \mathrm{Na}\left(\mathrm{MNa}^{+}\right)$: 331.0941, found 331.0934.

$(1 R, \quad 4 R, \quad 5 R)-3-((E)-2-(B e n z o[b]$ thiophen-2-yl)vinyl)-1,4dihydroxy-cyclohex-2-en-1,5-carbolactone (14b)
The experimental procedure used was the same as for diol 14a 60 utilizing silyl ether $\mathbf{1 3 b}(315 \mathrm{mg}, 0.58 \mathrm{mmol})$. Yield $=79 \mathrm{mg}$ $(43 \%) .[\alpha]_{20}^{\mathrm{D}}=-82.4^{\circ}(c 1.0$ in $\mathrm{MeOH}) ; \delta_{\mathrm{H}}\left(250 \mathrm{MHz} ; \mathrm{CD}_{3} \mathrm{OD}\right)$ : 7.78-7.67 (2 H, m, 2×ArH), 7.31-7.17 (3 H, m, 3×ArH), 7.22 (1 $\mathrm{H}, \mathrm{d}, J=16.0 \mathrm{~Hz}, \operatorname{ArCH}=\mathrm{CH}), 6.56(1 \mathrm{H}, \mathrm{d}, J=16.0 \mathrm{~Hz}$, $\mathrm{ArCH}=\mathrm{CH}), 6.14$ (1 H, s, H-2), $4.71(1 \mathrm{H}, \mathrm{m}, \mathrm{H}-5), 4.49(1 \mathrm{H}, \mathrm{d}$, $\left.{ }_{65} J=3.3 \mathrm{~Hz}, \mathrm{H}-4\right)$ and $2.44-2.35\left(2 \mathrm{H}, \mathrm{m}, \mathrm{CH}_{2}\right) ; \delta_{\mathrm{C}}(63 \mathrm{MHz}$; $\mathrm{CD}_{3} \mathrm{OD}$ ): 178.2 (C), 143.7 (C), 141.6 (C), 140.4 (C), 138.0 (C), $135.6(\mathrm{CH}), 129.9(\mathrm{CH}), 126.1(2 \times \mathrm{CH}), 125.7(\mathrm{CH}), 125.2(\mathrm{CH})$, $124.6(\mathrm{CH}), 123.1(\mathrm{CH}), 78.0(\mathrm{CH}), 74.5(\mathrm{C}), 65.8(\mathrm{CH})$ and 37.6 $\left(\mathrm{CH}_{2}\right) ; v_{\max }(\mathrm{film}) / \mathrm{cm}^{-1} 3427(\mathrm{OH})$ and $1780(\mathrm{CO}) ; \mathrm{MS}(\mathrm{ESI}) \mathrm{m} / \mathrm{z}$ $70=337\left(\mathrm{MNa}^{+}\right)$; HRMS (ESI) calcd for $\mathrm{C}_{17} \mathrm{H}_{14} \mathrm{O}_{4} \mathrm{SNa}\left(\mathrm{MNa}^{+}\right)$: 337.0505, found 337.0494.

$(1 R, 4 R, 5 R)-1,4,5-$ Trihydroxy-3-((E)-2-(naphth-2yl)vinyl)cyclohex-2-ene-1-carboxylic acid (5a)

75 A solution of the lactone 14a (30 mg, $0.10 \mathrm{mmol})$ in THF (1 mL) and aqueous lithium hydroxide $(0.5 \mathrm{~mL}, 0.25 \mathrm{mmol}, 0.5 \mathrm{M})$ was stirred at room temperature for $10 \mathrm{~min}$. Water was added and THF was removed under reduced pressure. The resulting aqueous solution was washed with diethyl ether $(\times 2)$ and the aqueous 80 extract was treated with Amberlite IR-120 until pH 6. The resin was filtered off and washed with Milli-Q water. The filtrate and the washings were lyophilised to give acid $\mathbf{5 a}(25 \mathrm{mg}, 77 \%)$ as a yellow solid. Mp: $197-199{ }^{\circ} \mathrm{C}$; $[\alpha]^{\mathrm{D}}{ }_{20}=-48.2^{\circ}(c 1.0$ in $\mathrm{MeOH})$; $\delta_{\mathrm{H}}\left(250 \mathrm{MHz} ; \mathrm{CD}_{3} \mathrm{OD}\right): 7.75-7.61(5 \mathrm{H}, \mathrm{m}, 5 \times \mathrm{ArH}), 7.37(2 \mathrm{H}$, $85 \mathrm{~m}, 2 \times \mathrm{ArH}), 7.02(1 \mathrm{H}, \mathrm{d}, J=16.3 \mathrm{~Hz}, \mathrm{ArCH}=\mathrm{CH}), 6.89(1 \mathrm{H}, \mathrm{d}, J$ $=16.3 \mathrm{~Hz}, \mathrm{ArCH}=\mathrm{CH}), 5.80(1 \mathrm{H}, \mathrm{s}, \mathrm{H}-2), 4.36(1 \mathrm{H}, \mathrm{d}, J=3.3$ $\mathrm{Hz}, \mathrm{H}-4), 3.96(1 \mathrm{H}, \mathrm{m}, \mathrm{H}-5)$ and $2.17\left(2 \mathrm{H}, \mathrm{m}, \mathrm{CH}_{2}\right) ; \delta_{\mathrm{C}}(63$ $\mathrm{MHz}$; $\left.\mathrm{CD}_{3} \mathrm{OD}\right)$ : 180.7 (C), 138.7 (C), 136.4 (C), 135.2 (C), 134.5 (C), $134.0(\mathrm{CH}), 130.9(\mathrm{CH}), 130.4(\mathrm{CH}), 129.2(\mathrm{CH}), 129.0$ $90(\mathrm{CH}), 128.6(\mathrm{CH}), 127.6(\mathrm{CH}), 127.3(\mathrm{CH}), 126.8(\mathrm{CH}), 124.5$ $(\mathrm{CH}), 74.3(\mathrm{C}), 71.6(\mathrm{CH}), 68.8(\mathrm{CH})$ and $35.9\left(\mathrm{CH}_{2}\right) ; v_{\max }$ $(\mathrm{KBr}) / \mathrm{cm}^{-1} 3410(\mathrm{OH})$ and $1651(\mathrm{CO}) ; \mathrm{MS}(\mathrm{ESI}) \mathrm{m} / \mathrm{z}=325$ $\left(\mathrm{M}-\mathrm{H}^{+}\right)$; HRMS (ESI) calcd for $\mathrm{C}_{19} \mathrm{H}_{17} \mathrm{O}_{5}\left(\mathrm{M}-\mathrm{H}^{+}\right)$: 325.1071 , found 325.1078 .

95

$(1 R, 4 R, 5 R)-3-((E)-2-(b e n z o[b]$ thiophen-2-yl)vinyl)-1,4,5trihydro-xycyclohex-2-ene-1-carboxylic acid (5b)

The experimental procedure used was the same as for acid 5a utilizing lactone 14b $(22.8 \mathrm{mg}, 0.07 \mathrm{mmol})$. Yield $=18.4 \mathrm{mg}$ $100(79 \%)$. Yellow solid. Mp: $184-185^{\circ} \mathrm{C} ;[\alpha]^{\mathrm{D}}{ }_{20}=-41.6^{\circ}(c 1.0$ in $\mathrm{MeOH}) ; \delta_{\mathrm{H}}\left(250 \mathrm{MHz} ; \mathrm{CD}_{3} \mathrm{OD}\right): 7.67(2 \mathrm{H}, \mathrm{m}, 2 \times \mathrm{ArH}), 7.22$ (4 $\mathrm{H}, \mathrm{m}, 3 \times \mathrm{ArH}+\mathrm{ArCH}=\mathrm{CH}), 6.63(1 \mathrm{H}, \mathrm{d}, J=16.0 \mathrm{~Hz}$, $\mathrm{ArCH}=\mathrm{CH}), 5.86(1 \mathrm{H}, \mathrm{s}, \mathrm{H}-2), 4.24(1 \mathrm{H}, \mathrm{d}, J=4.5 \mathrm{~Hz}, \mathrm{H}-4)$, $3.99(1 \mathrm{H}, \mathrm{m}, \mathrm{H}-5)$ and $2.14\left(2 \mathrm{H}, \mathrm{m}, \mathrm{CH}_{2}\right) ; \delta_{\mathrm{C}}(63 \mathrm{MHz}$; $\left.{ }_{105} \mathrm{CD}_{3} \mathrm{OD}\right)$ : $178.8(\mathrm{C}), 144.6(\mathrm{C}), 141.7(\mathrm{CH}), 140.3(\mathrm{C}), 140.2(\mathrm{C})$, $131.5(\mathrm{C}), 131.1(\mathrm{CH}), 125.9(\mathrm{CH}), 125.5(\mathrm{CH}), 124.7(\mathrm{CH})$, $124.5(\mathrm{CH}), 123.0(\mathrm{CH}), 123.1(\mathrm{CH}), 71.4(\mathrm{C}), 71.1(\mathrm{CH}), 37.9$ $\left(\mathrm{CH}_{2}\right)$ and $30.7(\mathrm{CH}) ; v_{\max }(\mathrm{KBr}) / \mathrm{cm}^{-1} 3435(\mathrm{OH}), 1639(\mathrm{CO})$; MS (ESI) $m / z=331\left(\mathrm{M}-\mathrm{H}^{+}\right)$; HRMS (ESI) calcd for $\mathrm{C}_{17} \mathrm{H}_{15} \mathrm{O}_{5} \mathrm{~S}$ $110\left(\mathrm{M}-\mathrm{H}^{+}\right): 331.0635$, found 331.0634 .

Reduction of 13a with Rosemund's catalyst: Preparation of $(1 R, 4 R, 5 R)-1,4-D i(t e r t-b u t y l d i m e t h y l s i l y l o x y)-3-(2-$ (naphthalen-2-yl)ethyl)cyclohex-2-en-1,5-carbolactone (15a) 115 and $\quad(\mathbf{1}, \mathbf{4 R}, \mathbf{5 R})-\mathbf{1 , 4 - d i ( t e r t - b u t y l d i m e t h y l s i l y l o x y ) - 3 - ( 2 - ~}$ $((5,6,7,8$-tetrahydronaphthalen-2-yl)ethyl)cyclohex-2-en-1,5- 


\section{carbolactone (15c)}

A suspension of alkene 13a (115 $\mathrm{mg}, 0.22 \mathrm{mmol})$ and Rosemund's catalyst (106 mg, $5 \%$ on weight) in a mixture of $50 \%$ THF/methanol $(10 \mathrm{~mL})$ was stirred under hydrogen 5 atmosphere at room temperature for $12 \mathrm{~h}$. The mixture was filtered over Celite and the residue was washed with methanol. The filtrate and washings were evaporated. The obtained residue was purified by flash chromatography on silica gel, eluting with (1:2) dichloromethane/hexanes to yield naphthyl derivative 15a 10 (89 mg, 75\%) and compound 15c (23 mg, 20\%). Data for 15a: White foam. $[\alpha]_{20}^{\mathrm{D}}=-6.5^{\circ}\left(c 1.0\right.$ in $\left.\mathrm{CHCl}_{3}\right) ; \delta_{\mathrm{H}}(250 \mathrm{MHz}$; $\left.\mathrm{CDCl}_{3}\right)$ : 7.86-7.80 (3 H, m, 3×ArH), $7.62(1 \mathrm{H}$, br s, $\mathrm{ArH})$, 7.56-7.45 $(2 \mathrm{H}, \mathrm{m}, 2 \times \mathrm{ArH}), 7.33(1 \mathrm{H}, \mathrm{dd}, J=8.4$ and $1.6 \mathrm{~Hz}$, ArH), 5.81 (1 H, s, H-2), 4.53 (1 H, m, H-5), $4.11(1 \mathrm{H}, \mathrm{d}, J=3.1$ $15 \mathrm{~Hz}, \mathrm{H}-4), 3.00-2.75\left(3 \mathrm{H}, \mathrm{m}, \mathrm{CH}_{2}+\mathrm{CHH}\right), 2.52-2.35(3 \mathrm{H}, \mathrm{m}$, $\left.\mathrm{CH}_{2}+\mathrm{CH} H\right), 0.99\left(9 \mathrm{H}, \mathrm{s}, \mathrm{C}\left(\mathrm{CH}_{3}\right)_{3}\right), 0.96\left(9 \mathrm{H}, \mathrm{s}, \mathrm{C}\left(\mathrm{CH}_{3}\right)_{3}\right), 0.21$ $\left(3 \mathrm{H}, \mathrm{s}, \mathrm{CH}_{3}\right), 0.18\left(6 \mathrm{H}, \mathrm{s}, 2 \times \mathrm{CH}_{3}\right)$ and $0.15\left(3 \mathrm{H}, \mathrm{s}, \mathrm{CH}_{3}\right) ; \delta_{\mathrm{C}}(63$ $\mathrm{MHz} ; \mathrm{CDCl}_{3}$ ): 176.2 (C), 138.6 (C), 138.4 (C), 133.7 (C), 132.1 (C), $131.4(\mathrm{CH}), 128.1(\mathrm{CH}), 127.6(\mathrm{CH}), 127.6(\mathrm{CH}), 127.0$ $20(\mathrm{CH}), 126.5(\mathrm{CH}), 126.0(\mathrm{CH}), 125.3(\mathrm{CH}), 76.0(\mathrm{CH}), 74.8(\mathrm{C})$,

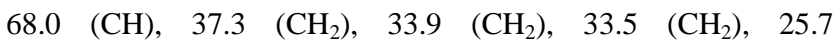
$\left(2 \times \mathrm{C}\left(\mathrm{CH}_{3}\right)_{3}\right), \quad 18.1 \quad\left(2 \times C\left(\mathrm{CH}_{3}\right)_{3}\right), \quad-3.0 \quad\left(2 \times \mathrm{CH}_{3}\right)$ and -4.5 $\left(2 \times \mathrm{CH}_{3}\right) ; v_{\max }(\mathrm{film}) / \mathrm{cm}^{-1} 1799(\mathrm{C}=\mathrm{O}) ; \mathrm{MS}(\mathrm{ESI}) \mathrm{m} / \mathrm{z}=561$ $\left(\mathrm{MNa}^{+}\right)$; HRMS (ESI) calcd for $\mathrm{C}_{31} \mathrm{H}_{46} \mathrm{O}_{4} \mathrm{Si}_{2} \mathrm{Na}\left(\mathrm{MNa}^{+}\right)$: ${ }_{25} 561.2829$, found 561.2823. Data for 15c: Colorless oil. $[\alpha]_{20}^{\mathrm{D}}=$ $-3.2^{\circ}$ (c 1.0 in $\left.\mathrm{CHCl}_{3}\right) ; \delta_{\mathrm{H}}\left(250 \mathrm{MHz} ; \mathrm{CDCl}_{3}\right): 7.09(1 \mathrm{H}, \mathrm{s}, \mathrm{ArH})$, $6.99(1 \mathrm{H}, \mathrm{d}, J=7.9 \mathrm{~Hz}, \operatorname{ArH}), 6.86(1 \mathrm{H}, \mathrm{m}, \operatorname{ArH}), 5.71(1 \mathrm{H}, \mathrm{s}$, $\mathrm{H}-2), 4.48$ (1 H, m, H-5), 4.04 (1 H, d, $J=3.1 \mathrm{~Hz}, \mathrm{H}-4), 2.74$ (7 $\left.\mathrm{H}, \mathrm{m}, 3 \times \mathrm{CH}_{2}+\mathrm{CHH}\right), 2.33\left(3 \mathrm{H}, \mathrm{m}, \mathrm{CH}_{2}+\mathrm{CHH}\right), 1.79(4 \mathrm{H}, \mathrm{m}$, $\left.{ }_{30} 2 \times \mathrm{CH}_{2}\right), 0.93\left(9 \mathrm{H}, \mathrm{s}, \mathrm{C}\left(\mathrm{CH}_{3}\right)_{3}\right), 0.92\left(9 \mathrm{H}, \mathrm{s}, \mathrm{C}\left(\mathrm{CH}_{3}\right)_{3}\right), 0.17$ (3 $\left.\mathrm{H}, \mathrm{s}, \mathrm{CH}_{3}\right), 0.16\left(3 \mathrm{H}, \mathrm{s}, \mathrm{CH}_{3}\right), 0.15\left(3 \mathrm{H}, \mathrm{s}, \mathrm{CH}_{3}\right)$ and $0.13(3 \mathrm{H}$, $\left.\mathrm{s}, \mathrm{CH}_{3}\right) ; \delta_{\mathrm{C}}\left(63 \mathrm{MHz} ; \mathrm{CDCl}_{3}\right): 176.2(\mathrm{C}), 138.7(\mathrm{C}), 138.1(\mathrm{C})$, $137.2(\mathrm{C}), 134.9(\mathrm{C}), 131.3(\mathrm{CH}), 129.3(\mathrm{CH}), 129.2(\mathrm{CH}), 125.5$ $(\mathrm{CH}), 76.1(\mathrm{CH}), 74.8(\mathrm{C}), 67.9(\mathrm{CH}), 37.4\left(\mathrm{CH}_{2}\right), 33.8\left(\mathrm{CH}_{2}\right)$, $3533.5\left(\mathrm{CH}_{2}\right), 29.5\left(\mathrm{CH}_{2}\right), 29.2\left(\mathrm{CH}_{2}\right), 25.8\left(\mathrm{C}\left(\mathrm{CH}_{3}\right)_{3}\right), 25.8$ $\left(\mathrm{C}\left(\mathrm{CH}_{3}\right)_{3}\right), \quad 25.7 \quad\left(3 \times \mathrm{CH}_{3}\right), \quad 23.4 \quad\left(\mathrm{CH}_{2}\right), \quad 23.4 \quad\left(\mathrm{CH}_{2}\right), \quad 18.0$ $\left(2 \times C\left(\mathrm{CH}_{3}\right)_{3}\right),-2.9\left(2 \times \mathrm{CH}_{3}\right),-4.4\left(\mathrm{CH}_{3}\right)$ and $-4.5\left(\mathrm{CH}_{3}\right) ; v_{\max }$ (film) $/ \mathrm{cm}^{-1} 1799$ (CO); MS (ESI) $\mathrm{m} / z=565\left(\mathrm{MNa}^{+}\right)$; HRMS (ESI) calcd for $\mathrm{C}_{31} \mathrm{H}_{50} \mathrm{O}_{4} \mathrm{Si}_{2} \mathrm{Na}\left(\mathrm{MNa}^{+}\right)$: 561.3140, found 40561.3128 .

\section{Reduction of 13a with Raney-Ni}

To a stirred solution of alkene 13a (932 mg, $1.74 \mathrm{mmol})$ in $50 \%$ $\mathrm{MeOH} / \mathrm{THF}(20 \mathrm{~mL})$ was treated with an aqueous suspension of ${ }_{45}$ Raney-Ni (aprox. 0.24 equivalents). The resulting suspension was deoxygenated and was stirred under hydrogen atmosphere at room temperature for $2.5 \mathrm{~h}$. The mixture was filtered over Celite and the residue was washed with methanol. The filtrate and washings were evaporated. The obtained residue was purified by 50 flash chromatography on silica gel, eluting with (5:95) acetone/hexanes to yield naphthyl derivative $\mathbf{1 5 a}$ (731 mg, 78\%).

$(1 R, 4 R, 5 R)-3-(2-(B e n z o[b]$ thiophen-2-yl)ethyl)-1,4-di(tertbutyl-dimethylsilyloxy)cyclohex-2-en-1,5-carbolactone (15b)

${ }_{55}$ The experimental procedure used was the same as for compound 15a using Rosemund's catalyst and utilizing alkene 13b (115.8 $\mathrm{mg}, 0.21 \mathrm{mmol})$. Yield $=65.2 \mathrm{mg}(56 \%)$. Colorless oil. $[\alpha]_{20}^{\mathrm{D}}=-$ $88.3^{\circ}$ (c 1.0 in $\left.\mathrm{CHCl}_{3}\right) ; \delta_{\mathrm{H}}\left(250 \mathrm{MHz} ; \mathrm{CDCl}_{3}\right): 7.77-7.65(2 \mathrm{H}, \mathrm{m}$,
$2 \times \mathrm{ArH}), 7.34-7.22(2 \mathrm{H}, \mathrm{m}, 2 \times \mathrm{ArH}), 6.98(1 \mathrm{H}, \mathrm{s}, \mathrm{ArH}), 5.78$ (1 $\left.{ }_{60} \mathrm{H}, \mathrm{s}, 1 \mathrm{H}, \mathrm{H}-2\right), 4.48$ (1 H, m, H-5), $4.07(1 \mathrm{H}, \mathrm{d}, J=3.1 \mathrm{~Hz}, \mathrm{H}-4)$, 3.07-3.00 (2 H, m, $\left.\mathrm{CH}_{2}\right), 2.47\left(2 \mathrm{H}, \mathrm{m}, \mathrm{CH}_{2}\right), 2.33(2 \mathrm{H}, \mathrm{m}$, $\left.\mathrm{CH}_{2}\right), 0.93\left(9 \mathrm{H}, \mathrm{s}, \mathrm{C}\left(\mathrm{CH}_{3}\right)_{3}\right), 0.90\left(9 \mathrm{H}, \mathrm{s}, \mathrm{C}\left(\mathrm{CH}_{3}\right)_{3}\right), 0.18(3 \mathrm{H}, \mathrm{s}$, $\left.\mathrm{SiCH}_{3}\right), 0.15\left(3 \mathrm{H}, \mathrm{s}, \mathrm{SiCH}_{3}\right), 0.12\left(3 \mathrm{H}, \mathrm{s}, \mathrm{SiCH}_{3}\right)$ and $0.09(3 \mathrm{H}$, s, $\left.\mathrm{SiCH}_{3}\right) ; \delta_{\mathrm{C}}\left(63 \mathrm{MHz} ; \mathrm{CDCl}_{3}\right): 176.0(\mathrm{C}), 144.9(\mathrm{C}), 137.7(\mathrm{C})$, $65131.7(\mathrm{CH}), 128.5(\mathrm{C}), 124.3(\mathrm{CH}), 123.7(\mathrm{CH}), 123.0(\mathrm{CH})$, $122.3(\mathrm{CH}+\mathrm{C}), 121.1(\mathrm{CH}), 76.1(\mathrm{CH}), 74.9(\mathrm{C}), 68.2(\mathrm{CH}), 37.3$

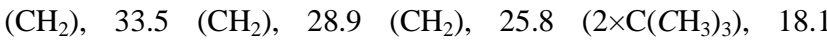
$\left(2 \times C\left(\mathrm{CH}_{3}\right)_{3}\right),-2.9\left(2 \times \mathrm{CH}_{3}\right)$, and $-4.4\left(2 \times \mathrm{CH}_{3}\right) ; \mathrm{v}_{\max }(\mathrm{film}) / \mathrm{cm}^{-1}$ 1799 (CO); MS (ESI) $\mathrm{m} / z=567\left(\mathrm{MNa}^{+}\right)$; HRMS (ESI) calcd for ${ }_{70} \mathrm{C}_{29} \mathrm{H}_{44} \mathrm{O}_{4} \mathrm{SSi}_{2} \mathrm{Na}\left(\mathrm{MNa}^{+}\right)$: 567.2391, found 567.2390.

$(1 R, 4 R, 5 R)-1,4-D i h y d r o x y-3-(2-(n a p h t h-2-y l) e t h y l) c y c l o h e x-2-$ en-1,5-carbolactone (16a)

The experimental procedure used was the same as for diol 14a 75 using silyl ether 15a (89 mg, $0.16 \mathrm{mmol})$. Yield = $39 \mathrm{mg}(79 \%)$. White foam. $[\alpha]_{20}^{\mathrm{D}}=-89.6^{\circ}(c 1.0$ in $\mathrm{MeOH}) ; \delta_{\mathrm{H}}(250 \mathrm{MHz}$; $\left.\mathrm{CD}_{3} \mathrm{OD}\right)$ : 7.76-7.69 (3 H, m, 3×ArH), 7.53 (1 H, br s, ArH), 7.37 $(2 \mathrm{H}, \mathrm{m}, 2 \times \mathrm{ArH}), 7.25(1 \mathrm{H}, \mathrm{dd}, J=8.4$ and $1.6 \mathrm{~Hz}, \mathrm{ArH}), 5.71$ (1 H, s, H-2), 4.59 (1 H, m, H-5), 4.05 (1 H, d, J=2.9 Hz, H-4), 80 2.92-2.79 (2 H, m, CH 2$), 2.55-2.42\left(2 \mathrm{H}, \mathrm{m}, \mathrm{CH}_{2}\right)$ and $2.28(2 \mathrm{H}$, $\left.\mathrm{m}, \mathrm{CH}_{2}\right) ; \delta_{\mathrm{C}}\left(63 \mathrm{MHz} ; \mathrm{CD}_{3} \mathrm{OD}\right): 179.0(\mathrm{C}), 140.6(\mathrm{C}), 140.1(\mathrm{C})$, $135.1(\mathrm{CH}), 133.5(\mathrm{C}), 131.3(\mathrm{CH}), 128.9(\mathrm{CH}), 128.5(2 \times \mathrm{CH})$, $128.2(\mathrm{CH}), 127.5(\mathrm{CH}), 126.8(\mathrm{CH}), 126.2(\mathrm{CH}), 77.9(\mathrm{CH})$, $73.9(\mathrm{C}), 67.6(\mathrm{CH}), 37.4\left(\mathrm{CH}_{2}\right)$ and $34.8\left(2 \times \mathrm{CH}_{2}\right) ; v_{\max }$ ${ }_{85}(\mathrm{KBr}) / \mathrm{cm}^{-1} 3448(\mathrm{OH})$ and 1776, 1761 and $1726(\mathrm{CO})$; MS (ESI) $m / z=333\left(\mathrm{MNa}^{+}\right)$; HRMS (ESI) calcd for $\mathrm{C}_{19} \mathrm{H}_{18} \mathrm{O}_{4} \mathrm{Na}\left(\mathrm{MNa}^{+}\right)$: 333.1097, found 333.1226 .

(1R,4R,5R)-3-(2-(Benzo[b]thiophen-2-yl)ethyl)-1,4-dihydroxy90 cyclohex-2-en-1,5-carbolactone (16b)

The experimental procedure used was the same as for diol 14a utilizing silyl ether $\mathbf{1 5 b}(65 \mathrm{mg}, 0.12 \mathrm{mmol})$. Yield $=22 \mathrm{mg}$ $(60 \%)$. White foam. $[\alpha]^{\mathrm{D}}{ }_{20}=-78.4^{\circ}(c 1.0$ in $\mathrm{MeOH}) ; \delta_{\mathrm{H}}(250$ $\left.\mathrm{MHz} ; \mathrm{CD}_{3} \mathrm{OD}\right)$ : $7.72(1 \mathrm{H}, \mathrm{m}, \mathrm{ArH}), 7.64$ (1 H, m, ArH), 95 7.11-7.28 (3 H, m, 3×ArH), $5.76(1 \mathrm{H}, \mathrm{s}, \mathrm{H}-2), 4.60(1 \mathrm{H}, \mathrm{m}, \mathrm{H}-$ 5), $4.01(1 \mathrm{H}, \mathrm{d}, J=3.1 \mathrm{~Hz}, \mathrm{H}-4), 3.10-3.02(1 \mathrm{H}, \mathrm{m}, \mathrm{CHH})$, 2.62-2.52 (3 H, m, $\left.\mathrm{CH}_{2}+\mathrm{CH} H\right)$ and $2.26\left(2 \mathrm{H}, \mathrm{m}, \mathrm{CH}_{2}\right) ; \delta_{\mathrm{C}}(63$ $\left.\mathrm{MHz} ; \mathrm{CD}_{3} \mathrm{OD}\right): 179.7$ (C), 146.5 (C), 141.8 (C), 140.4 (C), 131.0 $(\mathrm{CH}), 129.8(\mathrm{CH}), 125.6(\mathrm{CH}), 125.0(\mathrm{CH}), 124.4(\mathrm{CH}), 123.4$ $100(\mathrm{CH}+\mathrm{C}), 78.4(\mathrm{CH}), 74.4(\mathrm{C}), 68.0(\mathrm{CH}), 37.9\left(\mathrm{CH}_{2}\right), 35.0\left(\mathrm{CH}_{2}\right)$ and $29.9\left(\mathrm{CH}_{2}\right) ; v_{\max }(\mathrm{film}) / \mathrm{cm}^{-1} 3417(\mathrm{OH}), 1776$ and 1770 (CO); MS (ESI) $m / z=339\left(\mathrm{MNa}^{+}\right)$; HRMS (ESI) calcd for $\mathrm{C}_{17} \mathrm{H}_{16} \mathrm{O}_{4} \mathrm{SNa}\left(\mathrm{MNa}^{+}\right)$: 339.0662; found 339.0672.

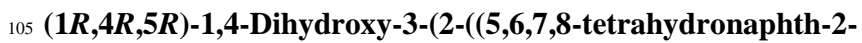
yl)ethyl)cyclohex-2-en-1,5-carbolactone (16c)

The experimental procedure used was the same as for diol 14a utilizing ether 15c (118 mg, $0.23 \mathrm{mmol})$. Yield: $65 \mathrm{mg}(90 \%)$. White solid. Mp: $155.6-156.4{ }^{\circ} \mathrm{C} ;[\alpha]_{20}^{\mathrm{D}}=-15.1^{\circ}$ (c 1.0 in $110 \mathrm{MeOH}) ; \delta_{\mathrm{H}}\left(250 \mathrm{MHz} ; \mathrm{CD}_{3} \mathrm{OD}\right): 7.00$ (1 H, s, ArH), 6.90-6.76 (2 H, m, 2×ArH), 5.67 (1 H, s, H-2), 4.59 (1 H, m, H-4), 4.03 (1 $\mathrm{H}, \mathrm{m}, \mathrm{H}-5), 2.68\left(7 \mathrm{H}, \mathrm{m}, 3 \times \mathrm{CH}_{2}+\mathrm{CHH}\right), 2.29(3 \mathrm{H}, \mathrm{m}$, $\left.2 \times \mathrm{CH}_{2}+\mathrm{CHH}\right)$ and $1.76-1.73\left(4 \mathrm{H}, \mathrm{m}, 2 \times \mathrm{CH}_{2}\right) ; \delta_{\mathrm{C}}(63 \mathrm{MHz}$; $\left.\mathrm{CD}_{3} \mathrm{OD}\right): 179.0(\mathrm{C}), 140.7$ (C), $139.4(\mathrm{C}), 137.8$ (C), $135.4(\mathrm{C})$, $115131.1(\mathrm{CH}), 130.0(2 \times \mathrm{CH}), 126.6(\mathrm{CH}), 77.9(\mathrm{CH}), 73.9(\mathrm{C}), 67.4$ $(\mathrm{CH}), 37.4\left(\mathrm{CH}_{2}\right), 35.1\left(\mathrm{CH}_{2}\right), 34.3\left(\mathrm{CH}_{2}\right), 30.2\left(\mathrm{CH}_{2}\right), 30.0$ 
$\left(\mathrm{CH}_{2}\right), 24.5\left(\mathrm{CH}_{2}\right)$ and $24.4\left(\mathrm{CH}_{2}\right) ; v_{\max }($ film $) / \mathrm{cm}^{-1} 3409(\mathrm{OH})$ and 1764 (CO); MS (ESI) $\mathrm{m} / z=337\left(\mathrm{MNa}^{+}\right)$; HRMS (ESI) calcd for $\mathrm{C}_{19} \mathrm{H}_{22} \mathrm{O}_{4} \mathrm{Na}\left(\mathrm{MNa}^{+}\right)$: 337.1410, found 337.1414.

5 (1R,4R,5R)-1,4-Dihydroxy-3-(2-(naphth-2-yl)ethyl)cyclohex-2ene-1-carboxylic acid (6a)

The experimental procedure used was the same as for acid 5a utilizing lactone 16a $(24.3 \mathrm{mg}, 0.08 \mathrm{mmol})$. Yield $=22 \mathrm{mg}$ (85\%). White solid. Mp: $120-122{ }^{\circ} \mathrm{C} ;[\alpha]^{\mathrm{D}}{ }_{20}=-8.3^{\circ}(c \quad 1.0$ in $10 \mathrm{MeOH}) ; \delta_{\mathrm{H}}\left(250 \mathrm{MHz} ; \mathrm{CD}_{3} \mathrm{OD}\right): 7.79-7.73(3 \mathrm{H}, \mathrm{m}, 3 \times \mathrm{ArH})$, 7.65 (1 H, br s, ArH), 7.43-7.35 (3 H, m, 3×ArH), $5.52(1 \mathrm{H}, \mathrm{s}$, $\mathrm{H}-2), 3.97-3.88$ (2 H, m, H-4+H-5), 3.06-3.98 (1 H, m, CHH), 2.91-2.82 (1 H, m, CHH), 2.75-2.68 (1 H, m, CHH), 2.49-2.41 $(1 \mathrm{H}, \mathrm{m}, \mathrm{CH} H)$ and $2.07\left(2 \mathrm{H}, \mathrm{m}, \mathrm{CH}_{2}\right) ; \delta_{\mathrm{C}}\left(63 \mathrm{MHz} ; \mathrm{CD}_{3} \mathrm{OD}\right)$ : 15178.3 (C), 145.0 (C), 140.8 (C), 135.1 (C), 133.5 (C), 128.8 $(\mathrm{CH}), 128.5(2 \times \mathrm{XH}), 128.3(\mathrm{CH}), 127.4(\mathrm{CH}), 126.8(\mathrm{CH}), 126.1$ $(\mathrm{CH}), 125.0(\mathrm{CH}), 74.9(\mathrm{CH}), 74.2(\mathrm{C}), 71.1(\mathrm{CH}), 40.3\left(\mathrm{CH}_{2}\right)$, $35.7\left(\mathrm{CH}_{2}\right)$ and $35.4\left(\mathrm{CH}_{2}\right) ; \mathrm{v}_{\max }($ film $) / \mathrm{cm}^{-1} 3435(\mathrm{OH})$ and 1720 (CO); MS (ESI) $m / z=327\left(\mathrm{M}-\mathrm{H}^{+}\right)$; HRMS (ESI) calcd for ${ }_{20} \mathrm{C}_{19} \mathrm{H}_{19} \mathrm{O}_{5}\left(\mathrm{M}-\mathrm{H}^{+}\right)$: 327.1227, found 327.1243.

$(1 R, 4 R, 5 R)-3-(2-(B e n z o[b]$ thiophen-2-yl)ethyl)-1,4-dihydroxycyclohex-2-ene-1,5-carboxylic acid $(6 \mathrm{~b})$

The experimental procedure used was the same as for acid 5a 25 utilizing lactone $\mathbf{1 6 b}(40 \mathrm{mg}, 0.13 \mathrm{mmol})$. Yield = $36 \mathrm{mg}(83 \%)$. White solild. Mp: $107-108{ }^{\circ} \mathrm{C} ;[\alpha]^{\mathrm{D}}{ }_{20}=-3.6^{\circ}(c 1.0$ in $\mathrm{MeOH}) ; \delta_{\mathrm{H}}$ (250 MHz; CD $\left.\mathrm{CD}_{3} \mathrm{OD}\right) 7.72(1 \mathrm{H}, \mathrm{d}, J=7.0 \mathrm{~Hz}, \mathrm{ArH}), 7.63(1 \mathrm{H}, \mathrm{d}$, $J=7.0 \mathrm{~Hz}, \mathrm{ArH}), 7.25-7.12(3 \mathrm{H}, \mathrm{m}, 3 \times \mathrm{ArH}), 5.43$ (1 H, s, H-2), 3.87 (2 H, m, H-5+H-4), $3.08(1 \mathrm{H}, \mathrm{m}, \mathrm{CH}), 2.60(1 \mathrm{H}, \mathrm{m}$, $\left.{ }_{30} \mathrm{CH} H\right)$ and $2.09\left(4 \mathrm{H}, \mathrm{m}, 2 \times \mathrm{CH}_{2}\right) ; \delta_{\mathrm{C}}\left(63 \mathrm{MHz} ; \mathrm{CD}_{3} \mathrm{OD}\right): 186.1$ (C), 146.7 (C), 141.7 (C), 140.7 (C), $140.0(\mathrm{CH}), 131.7$ (C), $125.0(\mathrm{CH}), 124.5(\mathrm{CH}), 123.8(\mathrm{CH}), 122.9(\mathrm{CH}), 122.0(\mathrm{CH})$, $78.0(\mathrm{CH}), 74.0(\mathrm{C}), 68.0(\mathrm{CH}), 40.6\left(\mathrm{CH}_{2}\right), 35.3\left(\mathrm{CH}_{2}\right)$ and 30.0 $\left(\mathrm{CH}_{2}\right) ; \mathrm{v}_{\max }(\mathrm{KBr}) / \mathrm{cm}^{-1} 3419(\mathrm{OH})$ and $1778(\mathrm{CO}) ; \mathrm{MS}(\mathrm{ESI}) \mathrm{m} / \mathrm{z}$, $35=333\left(\mathrm{M}-\mathrm{H}^{+}\right)$; HRMS (ESI) calcd for $\mathrm{C}_{17} \mathrm{H}_{17} \mathrm{O}_{5} \mathrm{~S}\left(\mathrm{M}-\mathrm{H}^{+}\right)$: 333.0791, found 333.0804.

$(1 R, 4 R, 5 R)-1,4,5$-Trihydroxy-3-(2-(5,6,7,8-tetrahydronaphth2-yl)ethyl)cyclohex-2-ene-1-carboxylic acid (6c)

${ }_{40}$ The experimental procedure used was the same as for acid 5a utilizing lactone $16 \mathrm{c}(34.5 \mathrm{mg}, 0.11 \mathrm{mmol})$. Yield $=31.2 \mathrm{mg}$ (85\%). Mp: $127-128^{\circ} \mathrm{C} ;[\alpha]^{\mathrm{D}_{20}}=-1.2^{\circ}(c 1.0$ in $\mathrm{MeOH}) ; \delta_{\mathrm{H}}(400$ $\left.\mathrm{MHz} ; \mathrm{CD}_{3} \mathrm{OD}\right): 6.90$ (2 H, m, 2×ArH), 6.87 (1 H, m, ArH), 5.45 (1 H, s, H-2), 3.89 (2 H, m, H-5+H-4), 2.78-2.74 (1 H, m, CHH), ${ }_{45} 2.71\left(4 \mathrm{H}, \mathrm{m}, 2 \times \mathrm{CH}_{2}\right), 2.63-2.57\left(2 \mathrm{H}, \mathrm{m}, \mathrm{CH}_{2}\right), 2.29(1 \mathrm{H}, \mathrm{m}$, $\mathrm{CH} H), 2.05\left(2 \mathrm{H}, \mathrm{m}, \mathrm{CH}_{2}\right)$, and $1.77\left(4 \mathrm{H}, \mathrm{m}, 2 \times \mathrm{CH}_{2}\right) ; \delta_{\mathrm{C}}(63$ $\left.\mathrm{MHz} ; \mathrm{CD}_{3} \mathrm{OD}\right)$ : $178.4(\mathrm{C}), 145.2$ (C), 140.2 (C), 137.8 (C), 135.3 (C), $122.9(2 \times \mathrm{CH}), 126.6(\mathrm{CH}), 124.7(\mathrm{CH}), 74.8(\mathrm{CH}), 74.3(\mathrm{C})$, $71.0(\mathrm{CH}), 40.3\left(\mathrm{CH}_{2}\right), 36.0\left(\mathrm{CH}_{2}\right), 34.9\left(\mathrm{CH}_{2}\right), 30.4\left(\mathrm{CH}_{2}\right), 30.0$ $50\left(\mathrm{CH}_{2}\right), 24.6\left(\mathrm{CH}_{2}\right)$, and $24.5\left(\mathrm{CH}_{2}\right) ; v_{\max }(\mathrm{KBr}) / \mathrm{cm}^{-1} 3427(\mathrm{OH})$, 1701 (CO); MS (ESI) $m / z=331\left(\mathrm{M}-\mathrm{H}^{+}\right)$; HRMS (ESI) calcd for $\mathrm{C}_{19} \mathrm{H}_{23} \mathrm{O}_{5}\left(\mathrm{M}-\mathrm{H}^{+}\right)$: 331.1540, found 331.1543.

\section{2-Vinylbenzo $[b]$ thiophene $(\mathbf{1 7 b})$}

${ }_{55}$ A Shlenck tube was charged with 2-bromobenzothiophene $(\mathbf{8 b})^{[10]}$ (250 mg, $\left.1.17 \mathrm{mmol}\right)$, vinylboronic acid pinacol ester $(0.3$ $\mathrm{mL}, 1.73 \mathrm{mmol}), \mathrm{Pd}\left(\mathrm{PPh}_{3}\right)_{4}(67 \mathrm{mg}, 0.06 \mathrm{mmol})$, aqueous $\mathrm{K}_{2} \mathrm{CO}_{3}$ $(3.45 \mathrm{~mL}, 1.1 \mathrm{M})$ and dioxane $(10 \mathrm{~mL})$. The resulting solution was heated at $100{ }^{\circ} \mathrm{C}$ for $5 \mathrm{~h}$. After cooling to room temperature, ${ }_{60}$ the reaction mixture was diluted with diethyl ether and water. The organic layer was separated and the aqueous phase was extracted with diethyl ether $(\times 3)$. The combined organic extracts were dried (anh. $\mathrm{Na}_{2} \mathrm{SO}_{4}$ ), filtered and concentrated under reduced pressure. The residue was purified by flash chromatography on silica gel, 65 eluting with (3:97) diethyl ether/hexanes to give 2vinylbenzo[b]thiophene $(\mathbf{1 7 b})^{[11]}(180 \mathrm{mg}, 96 \%)$.

\section{Preparation of 15a by $B$-alkyl Suzuki cross-coupling}

a) Preparation of borane 18a: A solution of 9-BBN-H $(5.7 \mathrm{~mL}$, $702.85 \mathrm{mmol}$, ca $0.5 \mathrm{M}$ in THF) was added to a flamed roundbottom flask under argon. After cooling to $0{ }^{\circ} \mathrm{C}$, 2vinylnaphthalene (17a) (200 mg, $1.29 \mathrm{mmol}$ ) was added. The mixture was warmed up slowly to room temperature and stirred for $3 \mathrm{~h}$ to give a solution of borane 18a.

75 b) B-alkyl Suzuki cross-coupling: To the borane solution obtained above, a solution of triflate $\mathbf{1 2}^{[2 \mathrm{c}]}(200 \mathrm{mg}, 0.37 \mathrm{mmol})$ in THF (4 $\mathrm{mL}), \mathrm{PdCl}_{2}$ (dppf) $(12.3 \mathrm{mg}, 0.02 \mathrm{mmol})$ and aqueous $\mathrm{K}_{3} \mathrm{PO}_{4}$ $(0.83 \mathrm{~mL}, 0.83 \mathrm{mmol}, 1 \mathrm{M})$ were added. The resultant solution was heated at $70{ }^{\circ} \mathrm{C}$ for $4 \mathrm{~h}$ under argon. After cooling to room 80 temperature, the solution was diluted with diethyl ether and water. The organic layer was separated and the aqueous phase was extracted with diethyl ether $(\times 2)$. The combined organic extracts were dried $\left(\mathrm{Na}_{2} \mathrm{SO}_{4}\right)$, filtered and concentrated under reduced pressure. The residue was purified by flash 85 chromatography on silica gel, eluting with (5:95) diethyl etherhexanes, to give compound $\mathbf{1 5 a}$ (156 mg, 80\%).

\section{Trimethyl(3-(naphth-2-yl)prop-1-ynyl)silane (19a)}

A two necks round bottom flask equipped with a condenser and a 90 pressure compensated addition funnel was charged with magnesium turnings (141 mg, $5.82 \mathrm{mmol}$ ) and a few iodine pellets. The system was flamed under vacuum and cooled under argon atmosphere. Dry THF (3 $\mathrm{mL}$ ) was added to the round bottom flask and the compensated addition funnel was charged 95 with a solution of 2-bromonaphthalene $(\mathbf{8 a})(1 \mathrm{~g}, 4.85 \mathrm{mmol})$ in dry THF $(5 \mathrm{~mL})$. This solution was slowly added to the suspension, which was heated under reflux for $2 \mathrm{~h}$. The reaction mixture was cooled to room temperature and then it was treated with a solution of 3-bromoprop-1-ynyl)trimethylsilane $(0.9 \mathrm{~mL}$, $1007.2 \mathrm{mmol})$ in dry THF (3 mL). The reaction mixture was heated under reflux for $2 \mathrm{~h}$ and then cooled to room temperature. Saturated $\mathrm{NH}_{4} \mathrm{Cl}$ was added and the organic layer was separated. The aqueous phase was extracted with dichloromethane $(\times 2)$. The combined organic extracts were dried ( $\mathrm{anh} . \mathrm{Na}_{2} \mathrm{SO}_{4}$ ), filtered and 105 concentrated under reduced pressure. Purification by flash chromatography on silica gel, using hexanes as eluent, gave alkyne 19a $(621 \mathrm{mg}, 54 \%)$ as a white solid. Mp: $61-63{ }^{\circ} \mathrm{C} ; \delta_{\mathrm{H}}$ (400 MHz; $\left.\mathrm{CDCl}_{3}\right): 7.85-7.80(4 \mathrm{H}, \mathrm{m}, 4 \times \mathrm{ArH}), 7.50-7.44(3 \mathrm{H}$, $\mathrm{m}, 3 \times \mathrm{ArH}), 3.82\left(2 \mathrm{H}, \mathrm{s}, \mathrm{CH}_{2}\right)$ and $0.24\left(9 \mathrm{H}, \mathrm{s}, 3 \times \mathrm{CH}_{3}\right) ; \delta_{\mathrm{C}}(100$ $\left.{ }_{110} \mathrm{MHz} ; \mathrm{CDCl}_{3}\right)$ : $133.8(\mathrm{C}), 133.5(\mathrm{C}), 132.3(\mathrm{C}), 128.1(\mathrm{CH}), 127.6$ $(2 \times \mathrm{CH}), 126.4(\mathrm{CH}), 126.2(\mathrm{CH}), 126.1(\mathrm{CH}), 125.5(\mathrm{CH}), 104.2$ (C), $87.2(\mathrm{C}), 26.4\left(\mathrm{CH}_{2}\right)$ and $0.11\left(3 \times \mathrm{CH}_{3}\right) ; v_{\max }(\mathrm{KBr}) / \mathrm{cm}^{-1}$ $2173(\mathrm{C} \equiv \mathrm{C})$.

115 (3-(Benzo[b]thiophen-3-yl)prop-1-ynyl)trimethylsilane (19d) The experimental procedure used was the same as for alkyne 19a 
utilizing 3-bromobenzo[b]thiophene (8d) (1 g, $4.7 \mathrm{mmol})$ and (3bromoprop-1-ynyl)trimethylsilane $(0.9 \mathrm{~mL}, 5.6 \mathrm{mmol})$. Yield = $791 \mathrm{mg}(69 \%)$. White solid. $\delta_{\mathrm{H}}\left(250 \mathrm{MHz} ; \mathrm{CDCl}_{3}\right): 7.90-7.86(1$ H, m, ArH), 7.76-7.74 (1 H, m, ArH), 7.45-7.37 (3 H, m, ArH), $53.81\left(2 \mathrm{H}, \mathrm{d}, J=1.25 \mathrm{~Hz}, \mathrm{CH}_{2}\right)$ and $0.26\left(9 \mathrm{H}, \mathrm{s}, 3 \times \mathrm{CH}_{3}\right) ; \delta_{\mathrm{C}}(63$ $\left.\mathrm{MHz} ; \mathrm{CDCl}_{3}\right): 140.6(\mathrm{C}), 137.9(\mathrm{C}), 130.6(\mathrm{C}), 124.3(\mathrm{CH}), 123.9$ $(\mathrm{CH}), 123.0(\mathrm{CH}), 122.9(\mathrm{CH}), 121.3(\mathrm{CH}), 102.9(\mathrm{C}), 87.3(\mathrm{C})$, $20.2\left(\mathrm{CH}_{2}\right)$ and $0.1\left(3 \times \mathrm{CH}_{3}\right) ; v_{\max }(\mathrm{KBr}) / \mathrm{cm}^{-1} 2179(\mathrm{C} \equiv \mathrm{C}) ; \mathrm{MS}$ (CI) $m / z=245\left(\mathrm{MH}^{+}\right)$.

10

\section{2-(Propa-1,2-dienyl)naphthalene (21a)}

A stirred solution of silyl ether $19 \mathrm{a}(30 \mathrm{mg}, 0.13 \mathrm{mmol})$ in methanol $(1.5 \mathrm{~mL})$ at $0{ }^{\circ} \mathrm{C}$ was treated with potassium carbonate (17 $\mathrm{mg}, 0.13 \mathrm{mmol})$. The ice bath was removed and the resulting 15 mixture was stirred for $1 \mathrm{~h}$. The reaction mixture was partitioned in water and diethyl ether. The organic layer was separated and the aqueous phase was extracted with diethyl ether $(\times 2)$. The combined organic extracts were dried (anh. $\left.\mathrm{Na}_{2} \mathrm{SO}_{4}\right)$, filtered and concentrated under reduced pressure. The residue was purified by

20 flash chromatography on silica gel, eluting with (10:90) diethyl ether/hexanes to give allene $\mathbf{2 1 a}(20 \mathrm{mg}, 91 \%)$ as a white solid. Mp: 55.7-56.3 ${ }^{\circ} \mathrm{C} ; \delta_{\mathrm{H}}\left(250 \mathrm{MHz} ; \mathrm{CDCl}_{3}\right): 7.43-7.36(3 \mathrm{H}, \mathrm{m}$, 3×ArH), 7.26 (1 H, s, ArH), 7.13-7.02 (3 H, m, 3×ArH), 5.96 (1 $\mathrm{H}, \mathrm{t}, J=6.25 \mathrm{~Hz}, \mathrm{CH})$ and $4.83\left(2 \mathrm{H}, \mathrm{d}, J=5.0 \mathrm{~Hz}, \mathrm{CH}_{2}\right) ; \delta_{\mathrm{C}}(75$ $25 \mathrm{MHz} ; \mathrm{CDCl}_{3}$ ): 210.5 (C), $133.8(\mathrm{C}), 132.7$ (C), 131.5 (C), 128.4 $(\mathrm{CH}), 127.9(\mathrm{CH}), 127.8(\mathrm{CH}), 126.4(\mathrm{CH}), 125.8(\mathrm{CH}), 125.5$ $(\mathrm{CH}), 124.8(\mathrm{CH}), 94.5(\mathrm{CH})$ and $79.2\left(\mathrm{CH}_{2}\right) ; \mathrm{MS}(\mathrm{CI}) \mathrm{m} / z=167$ [MH'] $\mathrm{MRMS}^{+}$; $\left.\mathrm{CI}\right)$ calcd for $\mathrm{C}_{13} \mathrm{H}_{11}\left(\mathrm{MH}^{+}\right)$: 167.0861 , found 167.0860 .

30

\section{2-(Prop-2-ynyl)naphthalene (20a)}

A stirred solution of silyl silane 19a $(600 \mathrm{mg}, 2.5 \mathrm{mmol})$ in ethanol $(11 \mathrm{~mL})$ was treated with a solution of $\mathrm{AgNO}_{3}$ in (2.3:1) $\mathrm{EtOH} / \mathrm{H}_{2} \mathrm{O}(11 \mathrm{~mL}, 0.35 \mathrm{M})$. The resultant solution was stirred on 35 the dark at room temperature for $2 \mathrm{~h}$ during which time a white solid was formed. An aqueous solution of potassium cyanide (3.3 $\mathrm{mL}, 7.6 \mathrm{M}$ ) was then added and the reaction mixture was stirred until disappearance of the white precipitate. Diethyl ether was added and the aqueous layer was separated. The organic extract 40 was washed with brine, dried (anh. $\mathrm{Na}_{2} \mathrm{SO}_{4}$ ), filtered and concentrated under reduced pressure. The residue was purified by flash chromatography on silica gel, using hexanes as eluent, to give alkyne 20a (297 mg, 72\%) as a white solid. Mp: $52-53{ }^{\circ} \mathrm{C}$; $\delta_{\mathrm{H}}\left(250 \mathrm{MHz} ; \mathrm{CDCl}_{3}\right): 7.84-7.81(4 \mathrm{H}, \mathrm{m}, 4 \times \mathrm{ArH}), 7.51-7.44$ (3 $\left.{ }_{45} \mathrm{H}, \mathrm{m}, 3 \times \mathrm{ArH}\right), 3.79\left(2 \mathrm{H}, \mathrm{d}, J=1.5 \mathrm{~Hz}, \mathrm{CH}_{2}\right)$ and $2.27(1 \mathrm{H}, \mathrm{t}, J$ $=1.8 \mathrm{~Hz}, \mathrm{C} \equiv \mathrm{CH}) ; \delta_{\mathrm{C}}\left(63 \mathrm{MHz} ; \mathrm{CDCl}_{3}\right): 133.4(2 \times \mathrm{C}), 132.3(\mathrm{C})$, $128.2(\mathrm{CH}), 127.6(2 \times \mathrm{CH}), 126.3(\mathrm{CH}), 126.2(\mathrm{CH}), 126.1(\mathrm{CH})$, $125.6(\mathrm{CH}), 81.9(\mathrm{C}), 70.7(\mathrm{CH})$ and $24.9\left(\mathrm{CH}_{2}\right) ; \mathrm{v}_{\max }(\mathrm{KBr}) / \mathrm{cm}^{-}$ ${ }^{1} 3282(\mathrm{C} \equiv \mathrm{C}) \mathrm{cm}^{-1}$. MS (CI) $\mathrm{m} / z=167\left(\mathrm{MH}^{+}\right)$; HRMS (CI) calcd ${ }_{50}$ for $\mathrm{C}_{13} \mathrm{H}_{11}\left(\mathrm{MH}^{+}\right)$: 167.0861, found 167.0858.

\section{3-(Prop-2-ynyl)benzo $[b]$ thiophene (20d)}

The experimental procedure used was the same as for alkyne 20a utilizing silyl ether 19d $(1.6 \mathrm{~g}, 6.5 \mathrm{mmol})$. Yield $=657 \mathrm{mg}(61 \%)$. 55 Yellow oil. $\delta_{\mathrm{H}}\left(250 \mathrm{MHz} ; \mathrm{CDCl}_{3}\right): 8.00(1 \mathrm{H}, \mathrm{m}, \mathrm{ArH}), 7.91(1 \mathrm{H}$, m, ArH), $7.79(2 \mathrm{H}, \mathrm{m}, 2 \times \operatorname{ArH}), 7.58(1 \mathrm{H}, \mathrm{s}, \mathrm{ArH}), 3.79(2 \mathrm{H}$, $\mathrm{dd}, J=2.8$ and $\left.1.3 \mathrm{~Hz}, \mathrm{CH}_{2}\right)$ and $2.29(1 \mathrm{H}, \mathrm{t}, J=2.8 \mathrm{~Hz}, \mathrm{C} \equiv \mathrm{CH})$; $\delta_{\mathrm{C}}\left(63 \mathrm{MHz} ; \mathrm{CDCl}_{3}\right): 140.1(\mathrm{C}), 138.5(\mathrm{C}), 131.3(\mathrm{C}), 124.6$
$(\mathrm{CH}), 124.3(\mathrm{CH}), 123.2(\mathrm{CH}), 122.8(\mathrm{CH}), 121.2(\mathrm{CH}), 80.6$ $60(\mathrm{CH}), 70.6(\mathrm{C})$ and $18.8\left(\mathrm{CH}_{2}\right) ; v_{\max }(\mathrm{film}) / \mathrm{cm}^{-1} 3293(\mathrm{C} \equiv \mathrm{C})$; MS (CI) $\mathrm{m} / \mathrm{z}=173\left(\mathrm{MH}^{+}\right)$; HRMS (CI) calcd for $\mathrm{C}_{11} \mathrm{H}_{9} \mathrm{~S}\left(\mathrm{MH}^{+}\right)$: 173.0425, found 173.0430 .

\section{2-Allylnaphthalene (22a)}

65 The experimental procedure used was the same as for alkyne 19a utilizing 2-bromonaphthalene (8a) (200 $\mathrm{mg}, 0.96 \mathrm{mmol})$ and allyl bromide $(0.09 \mathrm{~mL}, 1 \mathrm{mmol})$. Yield $=158 \mathrm{mg}(99 \%)$. Colorless oil. $\delta_{\mathrm{H}}\left(300 \mathrm{MHz} ; \mathrm{CDCl}_{3}\right): 7.91-7.85(3 \mathrm{H}, \mathrm{m}, 3 \times \mathrm{ArH}), 7.71$ (1 $\mathrm{H}, \mathrm{s}, \mathrm{ArH}), 7.58-7.40$ (3 H, m, 3×ArH), 6.23-6.07 (1 H, m, $\left.{ }_{70} \mathrm{CH}=\mathrm{CH}_{2}\right), 5.28-5.19\left(2 \mathrm{H}, \mathrm{m}, \mathrm{CH}=\mathrm{CH}_{2}\right)$ and $3.63(2 \mathrm{H}, \mathrm{d}, J=7.8$ $\left.\mathrm{Hz}, \mathrm{CH}_{2}\right) ; \delta_{\mathrm{C}}\left(75 \mathrm{MHz} ; \mathrm{CDCl}_{3}\right)$ : $137.5(\mathrm{C}), 137.3(\mathrm{CH}), 133.6$ (C), $132.1(\mathrm{C}), 127.9(\mathrm{CH}), 127.6(\mathrm{CH}), 127.4(\mathrm{CH}), 127.3(\mathrm{CH})$, $126.6(\mathrm{CH}), 125.9(\mathrm{CH}), 125.2(\mathrm{CH}), 116.0\left(\mathrm{CH}_{2}\right)$ and 40.3 $\left(\mathrm{CH}_{2}\right)$; MS (CI) $m / z=169\left(\mathrm{MH}^{+}\right)$; HRMS (CI) calcd for $\mathrm{C}_{13} \mathrm{H}_{13}$ $75\left(\mathrm{MH}^{+}\right): 169.1017$, found 169.1023 .

\section{3-Allylbenzo $[b]$ thiophene (22d)}

The experimental procedure used was the same as for 2allylnaphthalene (22a) utilizing 3-bromobenzo[b]thiophene $(\mathbf{8 d})$ $80(300 \mathrm{mg}, 1.4 \mathrm{mmol})$. Yield $=218 \mathrm{mg}(89 \%)$. Colorless oil. $\delta_{\mathrm{H}}$ $\left(250 \mathrm{MHz} ; \mathrm{CDCl}_{3}\right): 8.05-7.86(2 \mathrm{H}, \mathrm{m}, 2 \times \mathrm{ArH}), 7.58-7.38(3 \mathrm{H}$, $\mathrm{m}, 3 \times \mathrm{ArH}), 6.28-6.15\left(1 \mathrm{H}, \mathrm{m}, \mathrm{CH}=\mathrm{CH}_{2}\right), 5.36-5.27(2 \mathrm{H}, \mathrm{m}$, $\left.\mathrm{CH}=\mathrm{CH}_{2}\right)$ and $3.75\left(2 \mathrm{H}, \mathrm{m}, \mathrm{CH}_{2}\right) ; \delta_{\mathrm{C}}\left(63 \mathrm{MHz} ; \mathrm{CDCl}_{3}\right): 140.5$ (C), $138.8(\mathrm{C}), 135.5(\mathrm{CH}), 134.5(\mathrm{C}), 124.2(\mathrm{CH}), 123.8(\mathrm{CH})$, ${ }_{85} 122.8(\mathrm{CH}), 122.1(\mathrm{CH}), 121.8(\mathrm{CH}), 116.6\left(\mathrm{CH}_{2}\right)$ and 33.0 $\left(\mathrm{CH}_{2}\right)$; MS (CI) $m / z=175\left(\mathrm{MH}^{+}\right)$; HRMS $(\mathrm{CI})$ calcd for $\mathrm{C}_{11} \mathrm{H}_{11} \mathrm{~S}$ $\left(\mathrm{MH}^{+}\right)$: 175.0581, found 175.0582.

(1R,4R,5R)-1,4-Di(tert-butyldimethylsilyloxy)-3-(3-(naphth-290 yl)prop-1-ynyl)cyclohex-2-en-1,5-carbolactone (24a)

A Shlenck tube was charged with triflate $\mathbf{1 2}^{[2 \mathrm{c}]}(100 \mathrm{mg}, 0.19$ $\mathrm{mmol})$ and dry THF $(9.5 \mathrm{~mL})$. CuI (7.6 mg, $0.04 \mathrm{mmol})$, $\mathrm{Pd}\left(\mathrm{PPh}_{3}\right)_{4}$ (45 mg, $0.04 \mathrm{mmol}$ ), 2-(prop-2-ynyl)naphthalene (20a) (158 mg, $0.95 \mathrm{mmol})$ and piperidine $(0.25 \mathrm{~mL}, 2.47 \mathrm{mmol})$ were 95 added. The resultant solution was deoxygenated and heated at 40 ${ }^{\circ} \mathrm{C}$ for $4 \mathrm{~h}$. After cooling to room temperature, the reaction mixture was diluted with diethyl ether and water. The organic layer was separated and the aqueous phase was extracted with diethyl ether $(x 2)$. The combined organic extracts were washed 100 with saturated solution of sodium bicarbonate $(\times 2)$, dried (anh. $\mathrm{Na}_{2} \mathrm{SO}_{4}$ ), filtered and concentrated under reduced pressure. The residue was purified by flash chromatography on silica gel, eluting with a gradient of dichloromethane-hexanes (5:95 to $35: 65)$, to give naphthyl derivative $\mathbf{2 4 a}(102 \mathrm{mg}, 98 \%)$ as an 105 orange foam. $[\alpha]_{20}^{\mathrm{D}}=-136^{\circ}\left(c 1.0\right.$ in $\left.\mathrm{CHCl}_{3}\right) ; \delta_{\mathrm{H}}(250 \mathrm{MHz}$; $\left.\mathrm{CDCl}_{3}\right)$ : 7.84-7.77 (4 H, m, 4×ArH), 7.49-7.40 (3 H, m, 3×ArH), 6.27 (1 H, s, H-2), 4.49 (1 H, m, H-5), $4.17(1 \mathrm{H}, \mathrm{d}, J=3.3 \mathrm{~Hz}$, $\mathrm{H}-4), 3.88$ (2 H, s, $\left.\mathrm{CH}_{2} \mathrm{Ar}\right), 2.38\left(2 \mathrm{H}, \mathrm{m}, \mathrm{CH}_{2}-6\right), 0.92(9 \mathrm{H}, \mathrm{s}$, $\left.\mathrm{C}\left(\mathrm{CH}_{3}\right)_{3}\right), 0.89\left(9 \mathrm{H}, \mathrm{s}, \mathrm{C}\left(\mathrm{CH}_{3}\right)_{3}\right), 0.20\left(3 \mathrm{H}, \mathrm{s}, \mathrm{SiCH}_{3}\right), 0.16(3 \mathrm{H}$, $\left.110 \mathrm{~s}, \mathrm{SiCH}_{3}\right)$ and $0.12\left(6 \mathrm{H}, \mathrm{s}, 2 \times \mathrm{SiCH}_{3}\right) ; \delta_{\mathrm{C}}\left(63 \mathrm{MHz} ; \mathrm{CDCl}_{3}\right)$ : $175.0(\mathrm{C}), 140.7(\mathrm{CH}), 133.5(\mathrm{C}), 133.4(\mathrm{C}), 132.3(\mathrm{C}), 128.3$ $(\mathrm{CH}), 127.6(\mathrm{CH}), 127.6(\mathrm{CH}), 126.4(\mathrm{CH}), 126.3(\mathrm{CH}), 126.2$ $(\mathrm{CH}), 125.6(\mathrm{CH}), 127.8(\mathrm{C}), 89.5(\mathrm{C}), 80.4(\mathrm{C}), 75.8(\mathrm{CH}), 74.9$ (C), $68.2(\mathrm{CH}), 36.8\left(\mathrm{CH}_{2}\right), 25.8\left(\mathrm{CH}_{2}\right), 25.6\left(2 \times \mathrm{C}\left(\mathrm{CH}_{3}\right)_{3}\right), 18.0$ $115\left(2 \times C\left(\mathrm{CH}_{3}\right)_{3}\right),-3.1\left(2 \times \mathrm{SiCH}_{3}\right),-4.6\left(\mathrm{SiCH}_{3}\right)$ and $-4.9\left(\mathrm{SiCH}_{3}\right)$; $v_{\max }(\mathrm{KBr}) / \mathrm{cm}^{-1} 2225(\mathrm{C} \equiv \mathrm{C})$ and $1803(\mathrm{CO}) \mathrm{cm}^{-1}$; MS (ESI) $\mathrm{m} / \mathrm{z}$ 
$=571\left(\mathrm{MNa}^{+}\right)$; HRMS (ESI) calcd for $\mathrm{C}_{32} \mathrm{H}_{44} \mathrm{Si}_{2} \mathrm{O}_{4} \mathrm{Na}\left(\mathrm{MNa}^{+}\right)$: 571.2670, found 571.2664.

\section{(1R,4R,5R)-3-(3-(Benzo[b]thiophen-3-yl)prop-1-ynyl)-1,4-} ${ }_{5}$ di(tert-butyldimethylsilyloxy)cy-clohex-2-en-1,5-carbolactone (24d)

The experimental procedure used was the same as for naphthyl derivative 24a using alkyne $20 d$ (164 $\mathrm{mg}, 0.95 \mathrm{mmol}$ ) and triflate $12^{[2 \mathrm{c}]}(100 \mathrm{mg}, 0.19 \mathrm{mmol})$. Yield $=100 \mathrm{mg}(95 \%)$. Orange foam. ${ }_{10}[\alpha]_{20}^{\mathrm{D}}=-132^{\circ}\left(c 1.0\right.$ in $\left.\mathrm{CHCl}_{3}\right) ; \delta_{\mathrm{H}}\left(400 \mathrm{MHz} ; \mathrm{CDCl}_{3}\right): 7.87(1$ $\mathrm{H}, \mathrm{m}, \mathrm{ArH}), 7.75$ (1 H, m, ArH), 7.43-7.37 (2 H, m, 2×ArH), 7.35 (1 H, s, ArH), 6.26 (1 H, d, $J=1.6 \mathrm{~Hz}, \mathrm{H}-2), 4.48$ (1 H, dd, $J$ $=5.6$ and $3.2 \mathrm{~Hz}, \mathrm{H}-5), 4.14(1 \mathrm{H}, \mathrm{d}, J=3.2 \mathrm{~Hz}, \mathrm{H}-4), 3.87(2 \mathrm{H}$, s, $\left.\mathrm{CH}_{2} \mathrm{Ar}\right), 2.40\left(1 \mathrm{H}, \mathrm{d}, J=10.8 \mathrm{~Hz}, \mathrm{H}-6_{\mathrm{ax}}\right), 2.36(1 \mathrm{H}, \mathrm{ddd}, J=$ $1510.8,5.6$ and $\left.1.6 \mathrm{~Hz}, \mathrm{H}-6_{\mathrm{eq}}\right), 0.93\left(9 \mathrm{H}, \mathrm{s}, \mathrm{C}\left(\mathrm{CH}_{3}\right)_{3}\right), 0.88(9 \mathrm{H}, \mathrm{s}$, $\left.\mathrm{C}\left(\mathrm{CH}_{3}\right)_{3}\right), 0.20\left(3 \mathrm{H}, \mathrm{s}, \mathrm{SiCH}_{3}\right), 0.16\left(3 \mathrm{H}, \mathrm{s}, \mathrm{SiCH}_{3}\right), 0.09$ (3 H, s, $\left.\mathrm{SiCH}_{3}\right)$ and $0.07\left(3 \mathrm{H}, \mathrm{s}, \mathrm{SiCH}_{3}\right) ; \delta_{\mathrm{C}}\left(100 \mathrm{MHz} ; \mathrm{CDCl}_{3}\right): 174.9$ (C), $140.9(\mathrm{CH}), 140.6(\mathrm{C}), 137.9(\mathrm{C}), 130.1(\mathrm{C}), 124.5(\mathrm{CH})$, $124.1(\mathrm{CH}), 123.2(\mathrm{CH}), 122.9(\mathrm{CH}), 122.6(\mathrm{C}), 121.3(\mathrm{CH}), 88.2$ $20(\mathrm{C}), 80.2(\mathrm{C}), 75.8(\mathrm{CH}), 74.9(\mathrm{C}), 68.2(\mathrm{CH}), 36.8\left(\mathrm{CH}_{2}\right), 25.6$ $\left(2 \times \mathrm{C}\left(\mathrm{CH}_{3}\right)_{3}\right), 19.6\left(\mathrm{CH}_{2}\right), 18.0\left(2 \times C\left(\mathrm{CH}_{3}\right)_{3}\right),-3.1\left(2 \times \mathrm{SiCH}_{3}\right)$, $-4.7\left(\mathrm{SiCH}_{3}\right)$ and $-4.9\left(\mathrm{SiCH}_{3}\right) ; v_{\max }(\mathrm{KBr}) / \mathrm{cm}^{-1} 2227(\mathrm{C} \equiv \mathrm{C})$ and $1803(\mathrm{C}=\mathrm{O})$; MS (CI) $\mathrm{m} / z=555\left(\mathrm{MH}^{+}\right)$; HRMS (CI) calcd for $\mathrm{C}_{30} \mathrm{H}_{42} \mathrm{O}_{4} \mathrm{SSi}_{2} \mathrm{Na}\left(\mathrm{MNa}^{+}\right)$: 555.2408, found 555.2415.

25

(1R,4R,5R)-1,4-Di(tert-butyldimethylsilyloxy)-3-(3-(naphth-2yl)propyl)cyclohex-2-en-1,5-carbolactone (25a)

The experimental procedure used was the same as for compound 15a using alkyne 24a (168 mg, $0.30 \mathrm{mmol})$, Rosemund's catalyst $30(150 \mathrm{mg})$ and $50 \% \mathrm{THF} / \mathrm{methanol}(6 \mathrm{~mL})$. Purification by flash chromatography on silica gel, eluting with (30:70) dichloromethane/hexanes, gave saturated derivative 25a (166 mg, $98 \%)$ as a colorless oil. $[\alpha]^{\mathrm{D}}{ }_{20}=-49.1^{\circ}(c 1.0$ in $\mathrm{MeOH}) ; \delta_{\mathrm{H}}(400$ $\left.\mathrm{MHz} ; \mathrm{CDCl}_{3}\right): 7.76-7.58(3 \mathrm{H}, \mathrm{m}, 3 \times \mathrm{ArH}), 7.47$ (1 H, br s, $\left.\mathrm{ArH}\right)$, $357.45-7.39$ ( $2 \mathrm{H}, \mathrm{m}, 2 \times \mathrm{ArH}), 7.29(2 \mathrm{H}, \mathrm{dd}, J=1.6$ and $8.4 \mathrm{~Hz}$, $2 \times \mathrm{ArH}), 5.73(1 \mathrm{H}, \mathrm{d}, J=1.6 \mathrm{~Hz}, \mathrm{H}-2), 4.45(1 \mathrm{H}, \mathrm{m}, \mathrm{H}-5), 4.00$ $(1 \mathrm{H}, \mathrm{d}, J=3.2 \mathrm{~Hz}, \mathrm{H}-4), 2.85(2 \mathrm{H}, \mathrm{td}, J=1.6$ and $7.2 \mathrm{~Hz}$, $\left.\mathrm{CH}_{2} \mathrm{Ar}\right), 2.31\left(2 \mathrm{H}, \mathrm{m}, \mathrm{CH}_{2}\right), 2.06\left(2 \mathrm{H}, \mathrm{m}, \mathrm{CH}_{2}\right), 1.83(2 \mathrm{H}, \mathrm{m}$, $\left.\mathrm{CH}_{2}\right), 0.92\left(9 \mathrm{H}, \mathrm{s}, \mathrm{C}\left(\mathrm{CH}_{3}\right)_{3}\right), 0.85\left(9 \mathrm{H}, \mathrm{s}, \mathrm{C}\left(\mathrm{CH}_{3}\right)_{3}\right), 0.18(3 \mathrm{H}, \mathrm{s}$, $\left.{ }_{40} \mathrm{SiCH}_{3}\right), 0.14\left(3 \mathrm{H}, \mathrm{s}, \mathrm{SiCH}_{3}\right), 0.09\left(3 \mathrm{H}, \mathrm{s}, \mathrm{SiCH}_{3}\right)$ and $0.04(3 \mathrm{H}$, s, $\left.\mathrm{SiCH}_{3}\right) ; \delta_{\mathrm{C}}\left(100 \mathrm{MHz} ; \mathrm{CDCl}_{3}\right): 176.1(\mathrm{C}), 139.2(\mathrm{C}), 138.9(\mathrm{C})$, $133.6(\mathrm{C}), 132.0(\mathrm{C}), 130.7(\mathrm{CH}), 128.0(\mathrm{CH}), 127.6(\mathrm{CH}), 127.4$ $(\mathrm{CH}), 127.2(\mathrm{CH}), 126.5(\mathrm{CH}), 125.9(\mathrm{CH}), 125.1(\mathrm{CH}), 76.0$ $(\mathrm{CH}), 74.7(\mathrm{C}), 67.7(\mathrm{CH}), 37.2\left(\mathrm{CH}_{2}\right), 35.6\left(\mathrm{CH}_{2}\right), 31.4\left(\mathrm{CH}_{2}\right)$, $4528.5\left(\mathrm{CH}_{2}\right), 25.6\left(2 \times \mathrm{C}\left(\mathrm{CH}_{3}\right)_{3}\right), 18.0\left(\mathrm{C}\left(\mathrm{CH}_{3}\right)_{3}\right),-17.8\left(\mathrm{C}\left(\mathrm{CH}_{3}\right)_{3}\right)$, $-3.0\left(2 \times \mathrm{SiCH}_{3}\right),-4.6\left(\mathrm{SiCH}_{3}\right)$ and $-4.8\left(\mathrm{SiCH}_{3}\right) ; \mathrm{v}_{\max }(\mathrm{film}) / \mathrm{cm}^{-1}$ 1799 (CO); MS (ESI) $m / z=553\left(\mathrm{MH}^{+}\right)$; HRMS (ESI) calcd for $\mathrm{C}_{32} \mathrm{H}_{49} \mathrm{Si}_{2} \mathrm{O}_{4}\left(\mathrm{MH}^{+}\right): 553.3164$, found 553.3145.

(1R,4R,5R)-3-(3-(Benzo[b]thiophen-3-yl)propyl)-1,4-di(tert${ }_{50}$ butyldime-thylsilyloxy)cyclo-hex-2-en-1,5-carbolactone (25d) The experimental procedure used was the same as for saturated derivative 15a utilizing alkyne 24d $(60 \mathrm{mg}, 0.11 \mathrm{mmol})$. Yield = $60 \mathrm{mg}(98 \%)$. Yellow oil. $[\alpha]^{\mathrm{D}}{ }_{20}=-86.4^{\circ}\left(c 1.0\right.$ in $\left.\mathrm{CHCl}_{3}\right) ; \delta_{\mathrm{H}}$ $\left(250 \mathrm{MHz} ; \mathrm{CDCl}_{3}\right): 7.86(1 \mathrm{H}, \mathrm{d}, J=7.3 \mathrm{~Hz}, \mathrm{ArH}), 7.72(1 \mathrm{H}, \mathrm{d}$, $\left.{ }_{55} J=8.2 \mathrm{~Hz}, \mathrm{ArH}\right), 7.38(2 \mathrm{H}, \mathrm{m}, 2 \times \mathrm{ArH}), 7.07$ ( $\left.1 \mathrm{H}, \mathrm{s}, \mathrm{ArH}\right), 5.76$ $(1 \mathrm{H}, \mathrm{s}, \mathrm{H}-2), 4.48$ (1 H, m, H-5), $4.01(1 \mathrm{H}, \mathrm{d}, J=3.0 \mathrm{~Hz}, \mathrm{H}-4)$, $2.85\left(2 \mathrm{H}, \mathrm{t}, J=7.3 \mathrm{~Hz}, \mathrm{CH}_{2} \mathrm{Ar}\right), 2.32\left(2 \mathrm{H}, \mathrm{m}, \mathrm{CH}_{2}-6\right), 2.12(2 \mathrm{H}$, $\left.\mathrm{m}, \mathrm{CH}_{2}\right), 1.88\left(2 \mathrm{H}, \mathrm{m}, \mathrm{CH}_{2}\right), 0.93\left(9 \mathrm{H}, \mathrm{s}, \mathrm{C}\left(\mathrm{CH}_{3}\right)_{3}\right), 0.87(9 \mathrm{H}, \mathrm{s}$,
$\left.\mathrm{C}\left(\mathrm{CH}_{3}\right)_{3}\right), 0.19\left(3 \mathrm{H}, \mathrm{s}, \mathrm{SiCH}_{3}\right), 0.14\left(3 \mathrm{H}, \mathrm{s}, \mathrm{SiCH}_{3}\right), 0.10(3 \mathrm{H}, \mathrm{s}$, $\left.{ }_{60} \mathrm{SiCH}_{3}\right)$ and $0.04\left(3 \mathrm{H}, \mathrm{s}, \mathrm{SiCH}_{3}\right) ; \delta_{\mathrm{C}}\left(63 \mathrm{MHz} \mathrm{CDCl}_{3}\right): 176.1(\mathrm{C})$, $140.5(\mathrm{C}), 138.8(\mathrm{C}), 138.7(\mathrm{C}), 136.1(\mathrm{C}), 130.8(\mathrm{CH}), 124.1$ $(\mathrm{CH}), 123.9(\mathrm{CH}), 122.9(\mathrm{CH}), 121.6(\mathrm{CH}), 121.4(\mathrm{CH}), 75.9$ $(\mathrm{CH}), 74.7(\mathrm{C}), 67.7(\mathrm{CH}), 37.2\left(\mathrm{CH}_{2}\right), 31.2\left(\mathrm{CH}_{2}\right), 28.0\left(\mathrm{CH}_{2}\right)$,

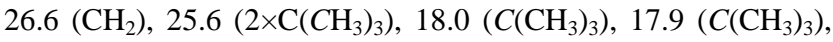
$651.0\left(\mathrm{SiCH}_{3}\right),-3.0\left(\mathrm{SiCH}_{3}\right)$, and $-4.6\left(\mathrm{SiCH}_{3}\right),-4.8\left(\mathrm{SiCH}_{3}\right) ; v_{\max }$ (film) $/ \mathrm{cm}^{-1} 1799(\mathrm{CO})$; MS (CI) $\mathrm{m} / \mathrm{z}=559\left(\mathrm{MH}^{+}\right)$.

$(1 R, 4 R, 5 R)-1,4-D i h y d r o x y-3-(3-(n a p h t h-2-y l) p r o p y l) c y c l o h e x-$ 2-en-1,5-carbolactone (26a)

70 The experimental procedure used was the same as for diol 14a utilizing silyl ether 25a (38 mg, $0.07 \mathrm{mmol}$ ). Purification by flash chromatography on silica gel, eluting with (1:1:1) diethyl ether/acetone/hexanes, gave diol 26a $(17 \mathrm{mg}, 77 \%)$ as a white foam. $[\alpha]^{\mathrm{D}}{ }_{20}=-151.4^{\circ}(c 1.0$ in $\mathrm{MeOH}) ; \mathrm{Mp}: 125-128{ }^{\circ} \mathrm{C} ; \delta_{\mathrm{H}}$ 75 (400 MHz; $\left.\mathrm{CD}_{3} \mathrm{OD}\right): 7.71(3 \mathrm{H}, \mathrm{m}, 3 \times \mathrm{ArH}), 7.53$ (1 H, br s, ArH), 7.36-7.23 (3 H, m, 3×ArH), $5.74(1 \mathrm{H}, \mathrm{s}, \mathrm{H}-2), 4.56(1 \mathrm{H}$, m, H-5), 3.98 (1 H, d, $J=4.0 \mathrm{~Hz}, \mathrm{H}-4), 2.67(2 \mathrm{H}, \mathrm{t}, J=7.0 \mathrm{~Hz}$, $\left.\mathrm{CH}_{2} \mathrm{Ar}\right), 2.27\left(2 \mathrm{H}, \mathrm{m}, \mathrm{CH}_{2}-6\right), 2.12\left(2 \mathrm{H}, \mathrm{m}, \mathrm{CH}_{2}\right)$ and $1.81(2 \mathrm{H}$, $\left.\mathrm{m}, \mathrm{CH}_{2}\right) ; \delta_{\mathrm{C}}\left(100 \mathrm{MHz} ; \mathrm{CD}_{3} \mathrm{OD}\right): 179.3(\mathrm{C}), 141.2(\mathrm{C}), 140.7$ $80(\mathrm{C}), 135.1(\mathrm{C}), 133.5(\mathrm{C}), 130.8(\mathrm{CH}), 128.9(\mathrm{CH}), 128.6(\mathrm{CH})$, $128.5(\mathrm{CH}), 128.3(\mathrm{CH}), 127.5(\mathrm{CH}), 126.9(\mathrm{CH}), 126.1(\mathrm{CH})$, $78.0(\mathrm{CH}), 74.0(\mathrm{C}), 67.6(\mathrm{CH}), 37.5\left(\mathrm{CH}_{2}\right), 36.3\left(\mathrm{CH}_{2}\right), 32.5$ $\left(\mathrm{CH}_{2}\right)$ and $29.7\left(\mathrm{CH}_{2}\right) ; v_{\max }(\mathrm{KBr}) / \mathrm{cm}^{-1} 3431(\mathrm{OH}), 3290(\mathrm{OH})$ and 1757 (CO); MS (ESI) $m / z=323\left(\mathrm{M}-\mathrm{H}^{+}\right)$; HRMS (ESI) calcd ${ }_{85}$ for $\mathrm{C}_{20} \mathrm{H}_{19} \mathrm{O}_{4}\left(\mathrm{M}-\mathrm{H}^{+}\right)$: 323.1278, found 323.1287.

\section{(1R,4R,5R)-3-(3-(Benzo[b]thiophen-3-yl)propyl)-1,4-} dihydroxy-cyclohex-2-en-1,5-carbolactone (26d)

The experimental procedure used was the same as for diol 14a 90 utilizing silyl ether 25d ( $85 \mathrm{mg}, 0.15 \mathrm{mmol}$ ). Yield: $33 \mathrm{mg}(67 \%)$. White solid. Mp: $156-160{ }^{\circ} \mathrm{C} .[\alpha]^{\mathrm{D}}{ }_{20}=-128.7^{\circ}(c 1.0$ in $\mathrm{MeOH})$; $\delta_{\mathrm{H}}\left(500 \mathrm{MHz} ; \mathrm{CD}_{3} \mathrm{OD}\right): 7.80(1 \mathrm{H}, \mathrm{d}, J=8.0 \mathrm{~Hz}, \mathrm{ArH}), 7.70(1$ $\mathrm{H}, \mathrm{d}, J=8.0 \mathrm{~Hz}, \mathrm{ArH}), 7.35-7.27(2 \mathrm{H}, \mathrm{m}, 2 \times \mathrm{ArH}), 7.16(1 \mathrm{H}, \mathrm{s}$, ArH), 5.79 (1 H, s, H-2), 4.59 (1 H, m, H-5), 4.01 (1 H, d, J=3.5 $\left.{ }_{95} \mathrm{~Hz}, \mathrm{H}-4\right), 2.78$ (2 H, m, $\left.\mathrm{CH}_{2} \mathrm{Ar}\right), 2.32-2.27\left(2 \mathrm{H}, \mathrm{m}, \mathrm{CH}_{2}-6\right)$, 2.25-2.21 (2 H, m, $\left.\mathrm{CH}_{2}\right), 1.97(1 \mathrm{H}, \mathrm{m}, \mathrm{CHH})$ and 1.86-1.77 (1 $\mathrm{H}, \mathrm{m}, \mathrm{CH} H) ; \delta_{\mathrm{C}}(63 \mathrm{MHz}$; acetone- $d 6)$ : $178.6(\mathrm{C}), 141.3(2 \times \mathrm{C})$, $140.8(\mathrm{C}), 138.3(\mathrm{C}), 131.7(\mathrm{CH}), 126.0(\mathrm{CH}), 125.7(\mathrm{CH}), 124.5$ $(\mathrm{CH}), 123.5(\mathrm{CH}), 123.2(\mathrm{CH}), 78.0(\mathrm{CH}), 74.6(\mathrm{C}), 68.4(\mathrm{CH})$, $10038.2\left(\mathrm{CH}_{2}\right), 33.4\left(\mathrm{CH}_{2}\right), 29.4\left(\mathrm{CH}_{2}\right)$ and $28.4\left(\mathrm{CH}_{2}\right) ; v_{\max }$ $(\mathrm{KBr}) / \mathrm{cm}^{-1} 2952(\mathrm{OH}), 2929(\mathrm{OH})$ and $1799(\mathrm{CO})$; MS (ESI) $\mathrm{m} / \mathrm{z}$ $=353\left(\mathrm{MNa}^{+}\right)$; HRMS (ESI) calcd for $\mathrm{C}_{18} \mathrm{H}_{18} \mathrm{O}_{4} \mathrm{SNa}\left(\mathrm{MNa}^{+}\right)$: 353.0823, found 353.0818 .

$105(\mathbf{1} R, \mathbf{4} R, \mathbf{5} R)-\mathbf{1}, \mathbf{4}, \mathbf{5}-$ Trihydroxy-3-(3-(naphth-2yl)propyl)cyclohex-2-ene-1-carboxylic acid (7a)

The experimental procedure used was the same as for acid 5a utilizing lactone 26a (30 mg, $0.09 \mathrm{mmol})$. Yield = $30 \mathrm{mg}(94 \%)$. White solid. $[\alpha]_{20}^{\mathrm{D}}=-23.2^{\circ}(c 1.0$ in $\mathrm{MeOH})$; $\mathrm{Mp}: 154-158{ }^{\circ} \mathrm{C}$; ${ }_{110} \delta_{\mathrm{H}}\left(400 \mathrm{MHz} ; \mathrm{CD}_{3} \mathrm{OD}\right): 7.67(3 \mathrm{H} \mathrm{m}, 3 \times \mathrm{ArH}), 7.54(1 \mathrm{H}$, br s, ArH), 7.30 (3 H, m, 3×ArH), 5.38 (1 H, s, H-2), 3.81 (2 H, m, H5+H-4), $2.70\left(2 \mathrm{H}, \mathrm{m}, \mathrm{CH}_{2} \mathrm{Ar}\right), 2.34(1 \mathrm{H}, \mathrm{m}, \mathrm{CHH})$ and

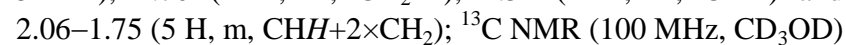
$\delta$ : $178.4(\mathrm{C}), 145.1(\mathrm{C}), 141.1(\mathrm{C}), 135.2(\mathrm{C}), 133.5(\mathrm{C}), 128.8$ $115(\mathrm{CH}), 128.6(\mathrm{CH}), 128.4(2 \times \mathrm{CH}), 127.6(\mathrm{CH}), 126.8(\mathrm{CH}), 126.1$ $(\mathrm{CH}), 124.9(\mathrm{CH}), 74.7(\mathrm{CH}), 74.3(\mathrm{C}), 71.1(\mathrm{CH}), 40.5\left(\mathrm{CH}_{2}\right)$, 
$36.3\left(\mathrm{CH}_{2}\right), 33.1\left(\mathrm{CH}_{2}\right)$ and $30.1\left(\mathrm{CH}_{2}\right) ; v_{\max }(\mathrm{KBr}) / \mathrm{cm}^{-1} 3390$ $(\mathrm{OH})$ and $1718(\mathrm{CO}) \mathrm{cm}^{-1}$. MS (ESI) $\mathrm{m} / z=341$ [M-H]; HRMS (ESI) calcd for $\mathrm{C}_{20} \mathrm{H}_{21} \mathrm{O}_{5}$ [M-H]: 341.1384, found 341.1384 .

\section{$5(1 R, 4 R, 5 R)-3-(3-(B e n z o[b]$ thiophen-3-yl)propyl)-1,4,5- trihydroxy-cyclohex-2-ene-1-carboxylic acid (7d)}

The experimental procedure used was the same as for acid 5a utilizing diol 26d (30 mg, $0.09 \mathrm{mmol})$. Yield = $26 \mathrm{mg}(87 \%)$. White solid. Mp: $118-119^{\circ} \mathrm{C} .[\alpha]^{\mathrm{D}}{ }_{20}=-34.1^{\circ}(c 1.0, \mathrm{MeOH}) .{ }^{1} \mathrm{H}$ $10 \mathrm{NMR}\left(400 \mathrm{MHz}, \mathrm{CD}_{3} \mathrm{OD}\right) \delta: 7.82(1 \mathrm{H}, \mathrm{d}, J=8.0 \mathrm{~Hz}, \mathrm{ArH}), 7.77$ $(1 \mathrm{H}, \mathrm{d}, J=7.2 \mathrm{~Hz}, \mathrm{ArH}), 7.36-7.27(2 \mathrm{H}, \mathrm{m}, 2 \times \mathrm{ArH}), 7.21(1 \mathrm{H}$, s, ArH), 5.47 (1 H, s, H-2), 3.91-3.84 (2 H, m, H-5+H-4), 2.95-2.79 (2 $\left.\mathrm{H}, \mathrm{m}, \mathrm{CH}_{2}\right), 2.54-2.42(1 \mathrm{H}, \mathrm{m}, \mathrm{CHH})$ and 2.18-1.80 (5 H, m, $\left.2 \times \mathrm{CH}_{2}+\mathrm{CH} H\right) ; \delta_{\mathrm{C}}\left(100 \mathrm{MHz} ; \mathrm{CD}_{3} \mathrm{OD}\right): 178.8$ $15(\mathrm{C}), 144.7(\mathrm{C}), 141.9(\mathrm{C}), 140.4(\mathrm{C}), 137.9(\mathrm{C}), 125.3(\mathrm{CH})$, $125.2(\mathrm{CH}), 124.9(\mathrm{CH}), 123.7(\mathrm{CH}), 122.8(\mathrm{CH}), 122.4(\mathrm{CH})$, $74.6(\mathrm{CH}), 74.4(\mathrm{C}), 71.1(\mathrm{CH}), 40.3\left(\mathrm{CH}_{2}\right), 33.4\left(\mathrm{CH}_{2}\right), 28.8$ $\left(\mathrm{CH}_{2}\right)$ and $28.2\left(\mathrm{CH}_{2}\right) ; \mathrm{U}_{\max }(\mathrm{KBr}) / \mathrm{cm}^{-1} 3367(\mathrm{OH})$ and 1709 (CO); MS (ESI) $m / z=347\left(\mathrm{M}-\mathrm{H}^{+}\right)$; HRMS (ESI) calcd for ${ }_{20} \mathrm{C}_{18} \mathrm{H}_{19} \mathrm{O}_{5} \mathrm{~S}\left(\mathrm{M}-\mathrm{H}^{+}\right)$: 347.0948, found 347.0955.

\section{Preparation of 25a by B-alkyl Suzuki cross-coupling}

a) Preparation of borane 23a: To a solution of 9-BBN-H (0.4 $\mathrm{mL}, 0.20 \mathrm{mmol}$, ca $0.5 \mathrm{M}$ in THF) in a flamed round-bottom

25 flask under argon 2-allylnaphthalene (22a) $(63 \mathrm{mg}, 0.37 \mathrm{mmol}$ ) was added. The mixture was stirred for $12 \mathrm{~h}$ to give a solution of borane 23a.

b) B-alkyl Suzuki cross-coupling: To the borane solution obtained above, $\mathrm{K}_{3} \mathrm{PO}_{4}(63 \mathrm{mg}, 0.28 \mathrm{mmol}), \mathrm{Pd}\left(\mathrm{PPh}_{3}\right)_{4}(33 \mathrm{mg}, 0.03$ $30 \mathrm{mmol})$, dioxane $(0.8 \mathrm{~mL})$ and triflate $\mathbf{1 2}^{[2 \mathrm{c}]}(100 \mathrm{mg}, 0.12 \mathrm{mmol})$ were added. The resultant solution was heated at $110^{\circ} \mathrm{C}$ for $12 \mathrm{~h}$ under argon. After cooling to room temperature, the solution was diluted with diethyl ether and water. The organic layer was separated and the aqueous phase was extracted with diethyl ether $35(\times 2)$. The combined organic extracts were dried $\left(\mathrm{Na}_{2} \mathrm{SO}_{4}\right)$, filtered and concentrated under reduced pressure. The residue was purified by flash chromatography on silica gel, eluting with a gradient of dichloromethane-hexanes (10:90 to 40:60), to give compound 25a (73 mg, 70\%).

40

Preparation of 25d by B-alkyl Suzuki cross-coupling

a) Preparation of borane 23d: To a solution of 9-BBN-H (0.44 $\mathrm{mL}, 0.22 \mathrm{mmol}$, ca $0.5 \mathrm{M}$ in THF) in a flamed round-bottom flask under argon 3-allylbenzo[b]thiophene (22d) (65 mg, 0.37 $45 \mathrm{mmol}$ ) was added. The mixture was stirred for $12 \mathrm{~h}$ to give a solution of borane $\mathbf{2 3 d}$.

b) B-alkyl Suzuki cross-coupling: To the borane solution obtained above, $\mathrm{K}_{3} \mathrm{PO}_{4}(84 \mathrm{mg}, 0.38 \mathrm{mmol}), \mathrm{Pd}\left(\mathrm{PPh}_{3}\right)_{4}(32 \mathrm{mg}, 0.03$ $\mathrm{mmol})$, dioxane $(0.8 \mathrm{~mL}), \mathrm{KBr}(25 \mathrm{mg}, 0.21 \mathrm{mmol})$ and triflate ${ }_{50} \mathbf{1 2}^{[2 \mathrm{c}]}(100 \mathrm{mg}, 0.12 \mathrm{mmol})$ were added. The resultant solution was heated at $110^{\circ} \mathrm{C}$ for $12 \mathrm{~h}$ under argon. After cooling to room temperature, the solution was diluted with diethyl ether and water. The organic layer was separated and the aqueous phase was extracted with diethyl ether $(\times 2)$. The combined organic 55 extracts were dried $\left(\mathrm{Na}_{2} \mathrm{SO}_{4}\right)$, filtered and concentrated under reduced pressure. The residue was purified by flash chromatography on silica gel, eluting with a gradient of dichloromethane-hexanes (10:90 to $40: 60)$, to give compound 25d (44 mg, 42\%).

60

\section{Dehydroquinase Assays}

The enzyme was purified and assayed as described previously. ${ }^{[12,2 \mathrm{~d}]}$

\section{${ }_{65}$ Docking studies}

They were carried out using program GOLD 5.0.1 ${ }^{[9]}$ and the enzyme geometries found in the crystal structure of the binary complex DHQ2-Hp/4c (PDB code: $2 \mathrm{WKS}^{[5 \mathrm{a}]}$ ) and DHQ2-Mt/ 4c (PDB code: $2 \mathrm{Y}^{\mathrm{I5b}]}$ ) In the latter case, not solved residues 18-20 70 were incorporated from the crystal structure of the fully resolved crystal structure of DHQ2-Mt in complex with $(1 R, 2 R, 4 S, 5 R)$ 1,4,5-trihydroxy-2-(4-methoxybenzyl)-3-

oxocyclohexanecarboxylic acid (PDB code: $2 X B 8^{[7]}$ ) The receptor was used as a dimer. Water molecules were removed 75 from all crystal structures with the expection of the water involved in the mechanism, which is located close to the carbonyl group of C3. Ligand geometries were minimized using the AM1 Hamiltonian as implemented in the program Gaussian $09^{[13]}$ and used as MOL2 files. Each ligand was docked in 25 independent 80 genetic algorithm (GA) runs, and for each of these a maximum number of $100000 \mathrm{GA}$ operations were performed on a single population of 50 individuals. Operator weights for crossover, mutation and migration in the entry box were used as default parameters $(95,95$, and 10 , respectively), as well as the hydrogen 85 bonding ( $4.0 \AA)$ and van der Waals $(2.5 \AA)$ parameters. The position of ligand $\mathbf{4 c}$ in both crystal structures was used to define the active-site and the radius was set to $7 \AA$. The "flip ring corners" flag was switched on, while all the other flags were off. The GOLD scoring function was used to rank the ligands in order 90 to fitness.

\section{Molecular dynamics simulations}

Ligand minimization. Ligand geometries were first refined by means of the semi-empirical quantum mechanical program ${ }_{95}$ MOPAC $^{[14]}$ using the AM1 Hamiltonian and PRECISE stopping criteria, and further optimised using a restricted Hartree-Fock (RHF) method and a 6-31G(d) basis set, as implemented in the $a b$ initio program Gaussian 09. ${ }^{[13]}$ The resulting wavefunctions were used to calculate electrostatic potential-derived (ESP) ${ }_{00}$ charges employing the restrained electrostatic potential (RESP) ${ }^{[15}$

] methodology, as implemented in the assisted model building with energy refinement (AMBER) ${ }^{[16]}$ suite of programs. The missing bonded and non-bonded parameters were assigned, by analogy or through interpolation from those already present in the 105 AMBER database (GAFF). ${ }^{[17,13]}$

Generation and minimization of the DHQ2-ligand complexes. Simulations were carried out using the enzyme geometries found in the crystal structure of DHQ2-Mt in complex $4 \mathbf{c}$ (PDB code $\left.{ }_{110} 2 \mathrm{Y} 1^{[5 \mathrm{~b}]}\right)$. Not solved residues $18-20$ were incorporated from the crystal structure of the fully resolved crystal structure of DHQ2$\mathrm{Mt}$ in complex with $(1 R, 2 R, 4 S, 5 R)-1,4,5$-trihydroxy-2-(4methoxybenzyl)-3-oxocyclo-hexanecarboxylic acid (PDB code: $\left.2 \mathrm{XB} 8^{[7]}\right)$. Taking into account that unfolding and refolding 115 studies of DHQ2 have shown that the trimer ${ }^{[18]}$ is the biological unit of the enzyme and on the basis of preliminary simulations on 
the monomer proving to be unstable under our simulation conditions, the trimer was used for these studies. Hydrogens were added to the protein using web-based PROPKA3.1 server, ${ }^{[19]}$ which assigned protonation states to all titratable residues at the 5 chosen $\mathrm{pH}$ of 7.0. However, $\delta$ and/or $\varepsilon$ protonation was manually corrected for His102 (dual) of the active site due to the mechanistic considerations and on the basis of results from preliminary MD simulations. Molecular mechanics parameters from the ff03 and GAFF force fields, respectively, were assigned 10 to the protein and the ligands using the LEaP module of AMBER 10.0. ${ }^{[20]}$ All terminal hydrogens were first minimizated in vacuo (2000 steps, half of them steepest descent, the other half conjugate gradient). Then, energy minimization using the implicit solvent GB model was carried out in stages, starting with ligand 15 (1000 steps, half of them steepest descent, the other half conjugate gradient), protein side-chains (1000 steps, idem) and finally the entire complex (1000 steps, idem). A positional restraint force constant of $50 \mathrm{kcal} \mathrm{mol}^{-1} \AA^{-2}$ to those unminimized atoms in each step was applied during all calculations. Thereafter 20 each refined DHQ2-ligand complex was neutralized by addition of sodium ions and immersed in a truncated octahedron of TIP3P water molecules. $^{[16,21,22]}$

Simulations. MD simulations were performed using the AMBER 2510.0 suite of programs and Amber ff03 force field. Periodic boundary conditions were applied and electrostatic interactions were treated using the smooth particle mesh Ewald method $(\mathrm{PME})^{[23]}$ with a grid spacing of $1 \AA$. The cutoff distance for the non-bonded interactions was $9 \AA$. SHAKE algorithm ${ }^{[24]}$ was 30 applied to all bonds containing hydrogen, using a tolerance of $10^{-}$

$5 \AA$ and an integration step of 2.0 fs. Minimization was carried out in three steps, starting with the octahedron water hydrogens, followed by solvent molecules and sodium counterions and finally the entire system. The minimized system was heated at $35300 \mathrm{~K}$ ( $1 \mathrm{~atm}, 25 \mathrm{ps}$, a positional restraint force constant of 50 kcal mol ${ }^{-1} \AA^{-2}$ ). These initial harmonic restraints were gradually reduced to $5 \mathrm{kcal} \mathrm{mol}^{-1} \AA^{-2}$ (10 steps) and the resulting systems were allowed to equilibrate further. MD with constraints of $5 \mathrm{kcal}$ $\mathrm{mol}^{-1} \AA^{-2}$ were carried out to all protein $\alpha$-carbons of the two 40 external subunits of the trimer and the beta sheets and alpha helix of the central subunit of the trimer for $10 \mathrm{~ns}$ (500 steps). System coordinates were collected every 2 ps for further analysis. Next, a slow-cooling MD simulation with constraints of $5 \mathrm{Kcal} \mathrm{mol}^{-1} \AA^{-2}$ was performed ( 6 steps until $273 \mathrm{~K}$ ). Finally, minimization of the 45 entire complexes was performed with constraints of $5 \mathrm{Kcal} \mathrm{mol}^{-1}$ $\AA^{-2}$

\section{Acknowledgements}

Financial support from the Xunta de Galicia (10PXIB2200122PR and GRC2010/12) and the Spanish Ministry of Science and 50 Innovation (SAF2010-15076) is gratefully acknowledged. BB, AS and AP thank the Spanish Ministry of Science and Innovation for FPU fellowships. We are also grateful to the Centro de Supercomputación de Galicia (CESGA) for the use of the Finis Terrae computer.

55

\section{Notes and References}

${ }^{a}$ Centro Singular de Investigación en Química Biológica y Materiales Moleculares (CIQUS), Universidad de Santiago de Compostela, calle Jenaro de la Fuente s/n, 15782 Santiago de Compostela, Spain. Fax: +34 60881 815704; Tel: +34 881 815726; E-mail:

concepcion.gonzalez.bello@usc.es

${ }^{b}$ Institute of Cell and Molecular Biosciences, Medical School, University of Newcastle upon Tyne, Catherine Cookson Building, Framlington Place, Newcastle upon Tyne NE2 4HH, UK.

$65{ }^{c}$ Departamento de Química Orgánica, Facultad de Química, Universidad de Santiago de Compostela, 15782 Santiago de Compostela, Spain.

$\dagger$ Electronic Supplementary Information (ESI) available: plots for inhibition data and extra data plots for Molecular dynamics simulations.

1 A. Koul, E. Arnoult, N. Lounis, J. Guillemont and K. Andries, Nature 2011, 469, 489-490.

2 a) C. González-Bello and L. Castedo, Med. Res. Rev. 2007, 27, 177-208. b) C. González-Bello, E. Lence, M. D. Toscano, L. Castedo, J. R. Coggins and C. Abell, J. Med. Chem. 2003, 46, 57355744; c) C. Sánchez-Sixto, V. F. V. Prazeres, L. Castedo, H. Lamb,

75 A. R. Hawkins and C. González-Bello, J. Med. Chem. 2005, 48, 4871-4881; d) V. F. V. Prazeres, C. Sánchez-Sixto, L. Castedo, H. Lamb, A. R. Hawkins, A. Riboldi-Tunnicliffe, J. R. Coggins, A. J. Lapthorn and C. González-Bello, ChemMedChem 2007, 2, 194-207; e) C. Sánchez-Sixto, V. F. V. Prazeres, L. Castedo, S. W. Suh, H. 80 Lamb, A. R. Hawkins, F. J. Cañada, J. Jiménez-Barbero and C. González-Bello, ChemMedChem 2008, 3, 756-770.

3 See also: a) M. Frederickson, E. J. Parker, A. R. Hawkins, J. R. Coggins and C. Abell, J. Org. Chem. 1999, 64, 2612-2613. b) M. Frederickson, A. W. Roszak, J. R. Coggins, A. J. Lapthorn and C. 85 Abell, Org. Biomol. Chem. 2004, 2, 1592-1596. c) M. D. Toscano, R. J. Payne, A. Chiba, O. Kerbarh and C. Abell, ChemMedChem 2007, 2, 101-112. d) R. J. Payne, F. Peyrot, O. Kerbarh, A. D. Abell and C. Abell, ChemMedChem 2007, 2, 1015-1029. e) R. J. Payne, A. Riboldi-Tunnicliffe, O. Kerbarh, A. D. Abell, A. J. Lapthorn and C. 90 Abell, ChemMedChem 2007, 2, 1010-1013. f) A. T. Tran, K. M. Cergol, N. P. West, E. J. Randall, W. J. Britton, S. A. I. Bokhari, M. Ibrahim, A. J. Lapthorn and R. J. Payne, ChemMedChem 2011, 6 , 262-265. g) M. V. B. Dias, W. C. Snee, K. M. Bromfield, E. J. Payne, S. K. Palaninathan, A. Ciulli, N. I. Howard, C. Abell, J. C. 95 Sacchettini and T. L. Blundell, Biochem. J. 2011, 436, 729-739.

4 a) J. Harris, C. González-Bello, C. Kleanthous, J. R. Coggins, A. R. Hawkins and C. Abell, Biochem. J. 1996, 319, 333-336. b) A. W. Roszak, D. A. Robinson, T. Krell, I. S. Hunter, M. Frederickson, C. Abell, J. R. Coggins and A. J. Lapthorn, Structure 2002, 10, 493-503.

5 a) V. F. V. Prazeres, L. Tizón, J. M. Otero, P. Guardado-Calvo, A. L. Llamas-Saíz, M. J. van Raaij, L. Castedo, H. Lamb, A. R. Hawkins and C. González-Bello, J. Med. Chem. 2010, 53, 191-200. b) L. Tizón, J. M. Otero, V. F. V. Prazeres, A. L. Llamas-Saíz, M. J. van 105 Raaij, H. Lamb, A. R. Hawkins, J. A. Ainsa, L. Castedo and C. González-Bello, J. Med. Chem. 2011, 54, 6063-6084.

6 This figure was prepared using PyMOL: DeLano, W.L. The PyMOL Molecular Graphics System. (2008) DeLano Scientific LLC, Palo Alto, CA, USA. http://www.pymol.org

1107 A. Peón, J. M. Otero, L. Tizón, V. F. V. Prazeres, A. L. Llamas-Saiz, G. C. Fox, M. J. van Raaij, H. Lamb, A. R. Hawkins, F. Gago, L. Castedo and C. González-Bello, ChemMedChem 2010, 5, $1726-1733$.

8 S. R. Chemler, D. Trauner and S. J. Danishefsky, Angew. Chem. Int. 115 Ed. 2001, 40, 4544-4568; Angew. Chem. 2001, 113, 4676-4701.

$9 \mathrm{http}: / /$ www.ccdc.cam.ac.uk/products/life sciencies/gold/

10 J. Fournier dit Chabert, B. Márquez, L. Neville, L. Joucla, S. Broussous, P. Bouhours, E. David, S. Pellet-Rostaing, B. Marquet, N. Moreau and M. Lemaire, Bioorg. Med. Chem. 2007, 15, 4482-4497.

12011 C. S. Bryan, J. A. Braunger and M. Lautens, Angew. Chem. Int. Ed. 2009, 48, 7064-7068; Angew. Chem. 2009, 121, 7198-7202.

12 D. G. Gourley, J. R. Coggins, N. W. Isaacs, J. D. Moore, I. G. Charles and A. R. Hawkins, J. Mol. Biol. 1994, 241, 488-491.

13 Gaussian 09, Revision A.2, M. J. Frisch, G. W. Trucks, H. B. Schlegel, G. E. Scuseria, M. A. Robb, J. R. Cheeseman, G. Scalmani, V. Barone, B. Mennucci, G. A. Petersson, H. Nakatsuji, M. Caricato, X. Li, H. P. Hratchian, A. F. Izmaylov, J. Bloino, G. Zheng, J. L. 
Sonnenberg, M. Hada, M. Ehara, K. Toyota, R. Fukuda, J. Hasegawa, M. Ishida, T. Nakajima, Y. Honda, O. Kitao, H. Nakai, T. Vreven, J. A. Montgomery, Jr., J. E. Peralta, F. Ogliaro, M. Bearpark, J. J. Heyd, E. Brothers, K. N. Kudin, V. N. Staroverov, R. Kobayashi, J.

$5 \quad$ Normand, K. Raghavachari, A. Rendell, J. C. Burant, S. S. Iyengar, J. Tomasi, M. Cossi, N. Rega, J. M. Millam, M. Klene, J. E. Knox, J. B. Cross, V. Bakken, C. Adamo, J. Jaramillo, R. Gomperts, R. E. Stratmann, O. Yazyev, A. J. Austin, R. Cammi, C. Pomelli, J. W. Ochterski, R. L. Martin, K. Morokuma, V. G. Zakrzewski, G. A.

10 Voth, P. Salvador, J. J. Dannenberg, S. Dapprich, A. D. Daniels, Ö. Farkas, J. B. Foresman, J. V. Ortiz, J. Cioslowski, and D. J. Fox, Gaussian, Inc., Wallingford CT, 2009.

14 J. Stewart, Aided Mol. Des. 1990, 4, 1-45.

15 a) W. D. Cornell, P. Cieplak, C. I. Bayly, I. R. Gould, K. M. Merz, D.

15 M. Ferguson, D. C. Spellmeyer, T. Fox, J. W. Caldwell, P. A. Kollman, J. Am. Chem. Soc. 1995, 117, 5179-5197. b) http://q4mdforcedfieldtools.org/RED/resp/

16 D. A. Case, T. E. Cheatham, T. Darden, H. Gohlke, R. Luo, K. M. Merz, O. Onufriev, C. Simmerling, B. Wang and R. J. Woods, J. Comput. Chem. 2005, 26, 1668-1688.

17 a) J. Wang, R. M. Wolf, J. W. Caldwell, P. A. Kollman and D. A. Case, J. Comp. Chem. 2004, 25, 1157-1174. b) J. Wang, W. Wang, P. A. Kollman and D. A. Case, J. Mol. Graph. Mod. 2006, 25, 247-260.

2518 N. C. Price, D. J. Boam, S. M. Kelly, D. Duncan, T. Krell, D. G. Gourley, J. R. Coggins, R. Virden and A. R. Hawkins, Biochem. J. 1999, 338, 195-202.

19 a) H. Li, A. D. Robertson and J. H. Jensen, Proteins 2005, 61, 704721. b) D. C. Bas, D. M. Rogers and J. H. Jensen, Proteins 2008, 73,

30 765-783. c) M. H. M. Olsson, C. R. Søndergard, M. Rostkowski, J. H. Jensen, J. Chem. Theor. Comp. 2011, 7, 525-537. d) C. R. Søndergard, M. H. M. Olsson, M. Rostkowski and J. H. Jensen, J. Chem. Theor. Comp. 2011, 7, 2284-2295.

20 Amber Tools 1.5: D. A. Case, T. A. Darden, T. E. Cheatham, III, C.

35 L. Simmerling, J. Wang, R. E. Duke, R. Luo, R. C. Walker, W. Zhang, K. M. Merz, B. Roberts, B. Wang, S. Hayik, A. Roitberg, G. Seabra, I. Kolossvai, K.F. Wong, F. Paesani, J. Vanicek, J. Liu, X. Wu, S. R. Brozell, T. Steinbrecher, H. Gohlke, Q. Cai, X. Ye, J. Wang, M.-J. Hsieh, G. Cui, D. R. Roe, D. H. Mathews, M. G. Seetin,

40 C. Sagui, V. Babin, T. Luchko, S. Gusarov, A. Kovalenko, P. A. Kollman (2010), AMBER 11, University of California, San Francisco.

21 J. Aqvist, J. Phys. Chem. 1990, 94, 8021-8024.

22 W. L. Jorgensen, J. Chandrasekhar and J. D. Madura, J. Chem. Phys. $45 \quad \mathbf{1 9 8 3}, 79,926-935$.

23 T. A. Darden, D. York and L. G. Pedersen, J. Chem. Phys. 1993, 98, 10089-10092.

24 J.-P. Ryckaert, G. Ciccotti and H. J. C. Berendsen, J. Comput. Phys. 1977, 23, 327-341. 